City University of New York (CUNY)

CUNY Academic Works

Dissertations, Theses, and Capstone Projects

CUNY Graduate Center

$2-2015$

\title{
Explicit Solutions of Imaginary Quadratic Norm Equations
}

Sandra Sze

Graduate Center, City University of New York

\section{How does access to this work benefit you? Let us know!}

More information about this work at: https://academicworks.cuny.edu/gc_etds/628

Discover additional works at: https://academicworks.cuny.edu

This work is made publicly available by the City University of New York (CUNY).

Contact: AcademicWorks@cuny.edu 


\title{
Explicit Solutions of Imaginary Quadratic Norm Equations
}

by

\author{
Sandra Sze
}

A dissertation submitted to the Graduate Faculty in Mathematics in partial fulfillment of the requirements for the degree of Doctor of Philosophy, The City University of New York.

2015 
(c) 2015

Sandra Sze

All Rights Reserved 
This manuscript has been read and accepted for the Graduate Faculty in Mathematics in satisfaction of the dissertation requirements for the degree of Doctor of Philosophy.

Victor Kolyvagin

Date

Chair of Examining Committee

Linda Keen

Date

Executive Officer

Victor Kolyvagin

Alexander Gamburd

Kenneth Kramer

Supervisory Committee

THE CITY UNIVERSITY OF NEW YORK 


\begin{abstract}
Explicit Solutions of Imaginary Quadratic Norm Equations
\end{abstract}

by

Sandra Sze

Advisor: Victor Kolyvagin

Let $K=\mathbb{Q}(\sqrt{-d})$ be an imaginary quadratic extension of $\mathbb{Q}$. Let $h$ be the class number and $D$ be the discriminant of the field $K$. Assume $p$ is a prime such that $\left(\frac{D}{p}\right)=1$. Then $p$ splits in $K$. The elements of the ring of integers $\mathscr{O}_{K}$ are of the form $x+\sqrt{-d} y$ if $d \equiv 1,2$ $(\bmod 4)$ and $x+\frac{1+\sqrt{-d}}{2} y$ if $d \equiv 3(\bmod 4)$, where $x$ and $y \in \mathbb{Z}$. The norm $N_{K / \mathbb{Q}}(x+\sqrt{-d} y)=x^{2}+d y^{2}$ and $N_{K / \mathbb{Q}}\left(x+\frac{1+\sqrt{-d}}{2} y\right)=\frac{(2 x+y)^{2}}{4}+\frac{d y^{2}}{4}$. In this thesis, we find the elements of norm $p^{h}$ explicitly. We also prove certain congruences for solutions of norm equations. 


\section{Acknowledgements}

I would like to first thank my advisor Victor Kolyvagin for coming up with the problem for this thesis, for his patience with me as I worked on it and for the many ideas he had in helping me solve this problem. I would also like to thank the NSF for their support of me through the RTG grant, as well as the National Physical Science Consortium for funding me during my first year at CUNY Graduate Center.

I thank the committee members Kenneth Kramer and Alexander Gamburd for taking the time to read my paper and providing feedback.

I am grateful for other professors who believed in my ability and helped me to learn mathematics: Lucien Szpiro, Jozef Dodziuk, Burton Randol, Raymond Hoobler, Delaram Kahrobaei, Carlos Moreno, Robert Thompson, the late Gilbert Baumslag, Roni Gouraige, Ravi Kulkarni, Wallace Goldberg, Russell Miller.

There are many other mathematicians who have helped me throughout the years. They were willing to talk math with me and helped me to become a better student and mathematician. Please forgive me if I forgot to add you to the list: Robert Suzzi-Valli, Ha Lam, Yunchun Hu, Viveka Erlandsson, Maggie Habeeb, Rebecca Steiner, Shlomo Ben-Har, 
Joey Hirsh, Phillip Williams, Nikita Miasnikov, Andrew Stout, Brian Stout, Lloyd West, Liang Zhao, Jorge Florez, Joe Kramer-Miller, John Basias, Ben Hutz, Adam Towsley, Blanca Marmolejo, Iryna Pavlyuk. I will always remember and cherish the hours we spent in the library, in empty classrooms, at Queens College, at different cafes, in the 8th floor cafeteria, working on homework, studying for the qualifiers and reviewing material our professors taught us.

I would like to thank my family and friends at Promise International Fellowship and at Resurrection Williamsburg. There are so many people at both congregations I would like to thank, so I'll only name pastors Timothy Harris and David Stancil as well as their wives Kim Harris and Mia Stancil. Thank you to all my friends who prayed with and for me and gave me encouragement during my years as a graduate student.

Other friends I would like to thank are Monica Chung, Kaisa Ercillo, Khadijah Rentas, Meiling Wang, Stuart Fontek, Joebrielle Basiao, Justin Bernstein, Michael Byrd, Sean Bryd, Ann Chow, Patrick Donnelly.

I would like to thank my family for their support of me as I pursued my career: my parents Jerry Sze and Huang Huang Li, my sisters Cindy and Tiffeny, my brother Allen, my thirteen cousins and my many aunts and uncles and my god sister Maria Mo. I would also like to thank my in-laws, especially Robert, Janet and Ted Williams, Rita Williams and Pam Jacobstein. Lastly, I would like to thank my husband Phillip Williams, mentioned above, who is one of my biggest supporter and an excellent mathematician, for his love, 
patience, guidance, and our shared love for yummy food and love for Jesus. 


\section{Contents}

1 Introduction 1

2 Preliminaries and Special Cases $\quad 8$

2.1 The Gauss Sums and the Stickelberger Theorem . . . . . . . . . . 8

2.2 Solutions of Norm Equations for $d=1,2,3 \ldots \ldots \ldots$

3 The General Case $\quad 22$

3.1 The Gross-Koblitz Formula . . . . . . . . . . . . . . . . . . . . 22

3.2 Estimates of Solutions of Norm Equations . . . . . . . . . . . . . 28

3.3 The Class Number Formulas . . . . . . . . . . . . . . . . . . 33

3.4 Representing an Element in $\mathbb{Q}(\sqrt{-d})$ with Norm $p^{h}$ as a Product of Gauss

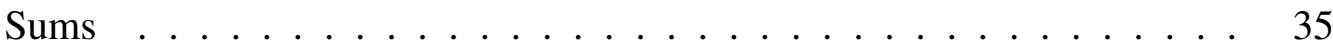

3.5 Solutions of Norm Equations in the case $\mathbb{Q}(\sqrt{-d}), d>0, d$ square-free,

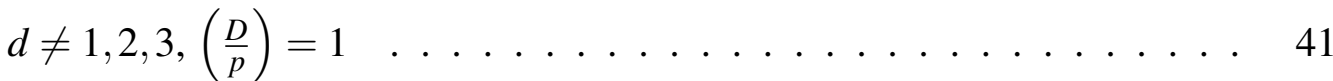


3.6 Solutions of Norm Equations as Normalized Trace of a Ratio of Products of Gauss Sums . . . . . . . . . . . . . . . . . . . . . 4 44

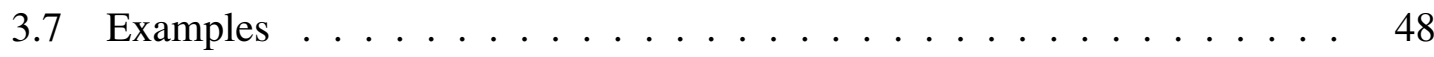

4 Finding the Height of the Stickelberger Ideal 51

Bibliography $\quad 56$ 


\section{Chapter 1}

\section{Introduction}

Let $K=\mathbb{Q}(\sqrt{-d})$ be an imaginary quadratic extension of $\mathbb{Q}$. Let $h$ be the class number and $D$ be the discriminant of the field $K$. (See Proposition 3.10 in section 3.3 for explicit formula for $h$.) Assume $p$ is a prime such that $\left(\frac{D}{p}\right)=1$. Then $p$ splits in $K$. The elements of the ring of integers $\mathscr{O}_{K}$ are of the form $x+\sqrt{-d} y$ if $d \equiv 1,2(\bmod 4)$ and $x+\frac{1+\sqrt{-d}}{2} y$ if $d \equiv 3(\bmod 4)$, where $x$ and $y \in \mathbb{Z}$. The norm $N_{K / \mathbb{Q}}(x+\sqrt{-d} y)=x^{2}+d y^{2}$ and $N_{K / \mathbb{Q}}\left(x+\frac{1+\sqrt{-d}}{2} y\right)=\frac{(2 x+y)^{2}}{4}+\frac{d y^{2}}{4}$. In this thesis, we find the elements of norm $p^{h}$ explicitly. That is, we find the general integer solutions $(x, y), p \not y$, to the equations

$$
\begin{aligned}
x^{2}+d y^{2} & =p^{h} \\
\frac{(2 x+y)^{2}}{4}+\frac{d y^{2}}{4} & =p^{h}
\end{aligned}
$$

for any imaginary quadratic field $K$ and prime $p$ such that $p$ splits in $K$.

We proceed as follows. By a theorem of Kronecker-Weber, $K$ is contained in a cyclo- 
tomic field $L=\mathbb{Q}\left(\zeta_{m}\right)$. We can choose $m$ to be $|D|$ if $d \neq 3, m=6$ if $d=3$.

We can find an element $Z \in \mathscr{O}_{K}$ such that the ideal $(Z)=\delta_{1}^{h}$, where $(p)=\delta_{1} \delta_{2}$ in $K$.

Let $\mathbb{F}_{q}$ be the residue field of a prime $\wp$ lying above $\delta_{1}$ in $L$ and $\omega$ a Teichmüller character of $\mathbb{F}_{q}$ for a prime $\mathfrak{p}$ lying above $\wp$ in $\mathbb{Q}\left(\zeta_{q-1}\right)$. Let $\tau_{p}(\chi)=-\sum_{a \in \mathbb{F}_{q}} \chi(a) \zeta_{p}^{\operatorname{Tr}(a)}$ be the Gauss sum associated to a character $\chi: \mathbb{F}_{q}^{\times} \rightarrow \mu_{q-1}, \chi(0)=0$.

Let $\chi=\omega^{\frac{q-1}{m}}$. Then $Z$ is a ratio $W / V$, where $W$ is a product of $\tau_{p}\left(\chi^{b_{i}}\right), b_{i} \in \mathbb{Z}$ and $V$ is a power of $p(d \neq 1,2,3)$ or a single Gauss sum $(d=1,2,3)$. We use Stickelberger relations, which give the prime decomposition of Gauss Sums, to find $Z$.

Let $x, y \in \mathbb{Z}$ such that $x+\sqrt{-d} y=Z$ if $d \equiv 1,2(\bmod 4), x+\frac{1+\sqrt{-d}}{2} y=Z$ if $d \equiv 3$ (mod 4). We use the Gross-Koblitz formula, which allows us to write Gauss Sums in terms of $p$-adic Gamma functions, to express the conjugate $\bar{Z}$ of $Z$ as a product of values of $p$-adic Gamma functions up to a sign. This implies an explicit congruence for $x$ or $2 x+y\left(\bmod p^{h}\right)$. Taking into account an estimate of the absolute value of $x$ or $2 x+y$ (see Proposition 3.6), this allows us to find $x$ or $2 x+y$ explicitly. We note that for the cases $d=1,2,3$, one can use the classical Stickelberger congruences instead of the GrossKoblitz formula. These cases were motivation for our study in the general case.

Let us describe our results. Let $N$ be a natural number, $t$ a rational $N$-integral number, then $\langle t\rangle_{N}$ denotes the integer such that $\langle t\rangle_{N} \equiv t(\bmod N)$ and $-\frac{N}{2}<\langle t\rangle_{N} \leq \frac{N}{2}$. We note that $h=1$ when $d=1,3,2$.

Let us first consider the case $d=1$. If $p \equiv 1(\bmod 4)$, then there exist, and are deter- 
mined up to a sign and transposition, $x, y \in \mathbb{Z}$ such that $x^{2}+y^{2}=p$. Let $\left(\begin{array}{l}n \\ r\end{array}\right)=\frac{n !}{r !(n-r) !}$ be a binomial coefficient. Let \langle\rangle$=\langle\rangle_{p}$.

We prove

Theorem 1.1. For a prime $p \equiv 1(\bmod 4)$

$$
\left\langle\frac{1}{2}\left(\begin{array}{c}
\frac{3 p-3}{4} \\
\frac{p-1}{4}
\end{array}\right)\right\rangle^{2}+\left\langle\frac{1}{2} \frac{\left(\frac{3 p-3}{p}\right) !}{\left(\frac{p-1}{4}\right) !}\right\rangle^{2}=p .
$$

This equality with \langle\rangle$=\langle\rangle_{n}$ characterizes primes equivalent to 1 modulo 4 among the naturals, which are not squares and are equivalent to 1 modulo 4.

Corollary 1.2. Let $p$ be a prime such that $p \equiv 1(\bmod 4)$ and $p=n^{2}+m^{2}$. Then

$$
\left(\begin{array}{c}
\frac{3 p-3}{4} \\
\frac{p-1}{4}
\end{array}\right) \quad(\bmod p) \in\{ \pm 2 n, \pm 2 m\} \quad(\bmod p) .
$$

Let $d=3$. If $p \equiv 1(\bmod 3)$ and $n^{2}+n m+m^{2}=p$, then the solutions of $x^{2}+x y+y^{2}=$ $p$ are

$$
\pm(n, m), \pm(-n, n+m), \pm(n+m,-n), \pm(n+m,-m), \pm(-n, n+m), \pm(m, n) .
$$

Therefore, the possible values for $2 x+y$ are

$$
\pm(2 n+m), \pm(n-m), \pm(n+2 m) \text {. }
$$

We prove 
Theorem 1.3. Let $p \equiv 1(\bmod 3), p \neq 7, \alpha=\left(\begin{array}{c}\frac{3 p-3}{6} \\ \frac{p-1}{6}\end{array}\right)$. Then

$$
\left(\langle\alpha\rangle, \sqrt{\frac{4 p-\langle\alpha\rangle^{2}}{3}}\right)
$$

is a solution in integers of the equation $x^{2}+3 y^{2}=4 p$.

Corollary 1.4. Let $p$ be a prime such that $p \equiv 1(\bmod 3)$ and $p=n^{2}+n m+m^{2}$. Then

$$
\left(\begin{array}{c}
\frac{3 p-3}{6} \\
\frac{p-1}{6}
\end{array}\right) \quad(\bmod p) \in\{ \pm(2 n+m), \pm(n-m), \pm(n+2 m)\} \quad(\bmod p)
$$

We also prove

Theorem 1.5. Let $p \equiv 1(\bmod 8)$ and $\alpha=\frac{1}{2}\left(\begin{array}{c}\frac{p-1}{2} \\ \frac{p-1}{8}\end{array}\right)$ or $p \equiv 3(\bmod 8)$ and $\alpha=\frac{1}{2}\left(\begin{array}{c}\frac{p-1}{2} \\ \frac{p-3}{8}\end{array}\right)$. Then

$$
\left(\langle\alpha\rangle, \sqrt{\frac{p-\langle\alpha\rangle^{2}}{2}}\right)
$$

is a solution in integers of the equation $x^{2}+2 y^{2}=p$.

Corollary 1.6. Suppose $p \equiv 1,3(\bmod 8)$ and $p=n^{2}+2 m^{2}$. Then

$$
\begin{aligned}
& \left(\begin{array}{c}
\frac{p-1}{2} \\
\frac{p-1}{8}
\end{array}\right) \equiv \pm 2 n \quad(\bmod p), \text { if } p \equiv 1 \quad(\bmod 8) \\
& \left(\begin{array}{l}
\frac{p-1}{2} \\
\frac{p-3}{8}
\end{array}\right) \equiv \pm 2 n \quad(\bmod p), \quad \text { if } p \equiv 3 \quad(\bmod 8)
\end{aligned}
$$

In fact, we first proved the congruences in the corollaries, which when combined with Proposition 3.6, imply Theorems 1.1, 1.3 and 1.5. 
Theorems 1.1, 1.3 and 1.5 will be proved in section 2.2 (Theorems 2.9, 2.10, 2.11), where we demonstrate our results and techniques for examples $d=1,3,2$.

For the case $d \neq 1,2,3$ we prove the following. Let $p$ be a prime such that $p$ splits in $K$, let $m=|D|$,

$$
\beta=\prod_{\substack{1 \leq a<m \\ \chi_{d}(a)=1}} \Gamma_{p}\left(\frac{a}{m}\right),
$$

where $\Gamma_{p}: \mathbb{Z}_{p} \rightarrow \mathbb{Z}_{p}^{\times}$is the $p$-adic Gamma function, $\chi_{d}:(\mathbb{Z} / m \mathbb{Z})^{\times} \rightarrow\{ \pm 1\}$ is the quadratic character associated to $K$ (see Proposition 3.10). For an $N$-integral $t$, let $[t]_{N}$ be the integer in the interval $[0, N)$ congruent to $t(\bmod N)$.

Let $c=c(d, p)$ be a natural number defined in Proposition 3.7. Note that if $k=k(h)=$ $\frac{h}{2}+1$ if $h$ is even, $k=\frac{h+1}{2}$ if $h$ is odd, then $c=k=h$ if $h \leq 2, c=k$ or $k+1$ or (if $p=2$ ) $k+2$ if $h>2, c=k$ if $d \equiv 1,2(\bmod 4) ; c=k$ if $d \equiv 3(\bmod 4), d$ is not a prime, $p \geq 5$; $c=k$ if $d \equiv 3(\bmod 4)$ is a prime and $p \geq 17$.

$$
\begin{gathered}
\text { Let } c \leq i \leq h, i \neq 2 \text { if } p=2,\langle\rangle=\langle\rangle_{p^{i}},[\quad]=[]_{p^{i}} \text { Let } T=\left[\frac{1}{m}\right], \\
\alpha=\prod_{\substack{1 \leq a<m \\
\chi_{d}(a)=1}}(-1)^{[a T]} \prod_{\substack{1 \leq j<[a T] \\
(j, p)=1}} j
\end{gathered}
$$

We prove in section 3.5,

Theorem 1.7. Let $d \neq 1,2,3$, $p$ be a prime that splits in $K, p \neq 2$ if $d=15, p \neq 5$ if $d=11$. 
Then for $d \equiv 1,2(\bmod 4)$,

$$
\begin{aligned}
& x=\left\langle\frac{1}{2} \alpha\right\rangle=\left\langle\frac{1}{2} \beta\right\rangle, \\
& y= \pm \sqrt{\frac{p^{h}-x^{2}}{d}}
\end{aligned}
$$

is a solution in $\mathbb{Z}, p \quad X y$, of the equation $x^{2}+d y^{2}=p^{h}$.

For $d \equiv 3(\bmod 4), x$ and $y$ defined by

$$
\begin{aligned}
2 x+y & =\langle\alpha\rangle=\langle\beta\rangle, \\
y & = \pm \sqrt{\frac{4 p^{h}-(2 x+y)^{2}}{d}}
\end{aligned}
$$

is a solution in $\mathbb{Z}, p \quad X y$, of the equation $(2 x+y)^{2}+d y^{2}=4 p^{h}$.

Corollary 1.8. If $p^{h}=n^{2}+d s^{2}$ or $p^{h}=\left(n+\frac{s}{2}\right)^{2}+d \frac{s^{2}}{4}$, with $n, s \in \mathbb{Z}, p \not s$, when $d \equiv 1,2$ $(\bmod 4)$ or $d \equiv 3(\bmod 4)$, respectively, then

$$
\alpha \equiv \beta \equiv \pm 2 n, \text { or } \pm(2 n+s) \quad\left(\bmod p^{i}\right)
$$

respectively.

Theorems 1.1, 1.3, 1.5 and 1.7 provide us with explicitly determined solutions of norm equations. They also imply the corresponding congruences modulo $p^{h}$. Theorems $1.1,1.3$, 1.5, 1.7 and their corollaries are the main results of this thesis.

We note that one can also express solutions $x, x+\frac{1}{2} y$ (and then find $y$ ) of the norm equations by directly applying the normalized trace to the element $Z$ or its image in $\mathbb{F}_{q}$ 
(see section 3.6 for the corresponding formulas).

Let us outline this thesis. In section 2.1, we describe the Stickelberger ideal and give the classical result about Gauss sums. Special cases $d=1,2,3$ are computed in section 2.2. In section 3.1, we give the Gross-Koblitz formula and use it to give the representation of Gauss sums in section 3.4. Section 3.2 contains estimates of solutions of norm equations. We introduce the element $Z$ and consider the cases $d=1,2,3$ in sections 3.4 and 3.5. In section 3.7, we demonstrate examples of computations using our formulas. In chapter 4, we find the height of the Stickelberger ideal. 


\section{Chapter 2}

\section{Preliminaries and Special Cases}

\subsection{The Gauss Sums and the Stickelberger Theorem}

Let $\zeta_{m}$ denote a primitive $m^{\text {th }}$ of unity and let $M / \mathbb{Q}$ be a finite abelian extension. By a theorem of Kronecker-Weber, $M \subset \mathbb{Q}\left(\zeta_{m}\right)$ for some $m$. We will assume that $m$ is minimal. Let $G=\operatorname{Gal}(M / \mathbb{Q})$, a quotient of $G_{m}=\operatorname{Gal}\left(\mathbb{Q}\left(\zeta_{m}\right) / \mathbb{Q}\right)=(\mathbb{Z} / m \mathbb{Z})^{\times}$.

For $(a, m)=1$, let $\sigma_{a}$ denote the element $\zeta_{m} \mapsto \zeta_{m}^{a}$ of $G_{m}$ as well as its restriction to $M$.

Definition 2.1. The Stickelberger element in the group-ring $\mathbb{Q}[G]$ is defined to be

$$
\theta=\theta(M)=\sum_{\substack{1 \leq a<m \\(a, m)=1}} \frac{a}{m} \sigma_{a}^{-1} .
$$

Then $I=I(M)=\mathbb{Z}[G] \cap \theta \mathbb{Z}[G]$ is an ideal of $\mathbb{Z}[G]$ consisting of $\mathbb{Z}[G]$ multiples of $\theta$ with coefficients in $\mathbb{Z}$. This ideal is called the Stickelberger ideal.

Lemma 2.2. If $M=\mathbb{Q}\left(\zeta_{m}\right)$ then $I=I^{\prime} \theta$, where $I^{\prime}$ is the ideal of $\mathbb{Z}[G]$ generated by all elements of the form $c-\sigma_{c}$ for $(c, m)=1$. 
Proof. See [7].

Definition 2.3. We define the action of $\mathbb{Z}[G]$ on ideals by: if $x=\sum x_{\sigma} \sigma \in \mathbb{Z}[G]$, then $x$ acts on ideals A of $M$ by

$$
A^{x}=\prod\left(A^{\sigma}\right)^{x_{\sigma}}
$$

We now give some definitions and properties of Gauss and Jacobi Sums.

Let $p$ be an odd prime and $q=p^{f}$. Let $\zeta_{p}$ be a fixed primitive $p$-th root of unity. The Galois group Gal $\left(\mathbb{F}_{q} / \mathbb{F}_{p}\right)$ is cyclic, generated by the Frobenius automorphism $\sigma_{p}: x \mapsto x^{p}$. Let $\lambda: \mathbb{F}_{q} \rightarrow \mathbb{C}^{\times}$be the additive character, defined by $\lambda(x)=\zeta_{p}^{\operatorname{Tr}(x)}$, where $\operatorname{Tr}(x)=$ $\operatorname{Tr}_{\mathbb{F}_{q} / \mathbb{F}_{p}}(x)=x+x^{p}+\cdots+x^{p^{f-1}}$ is the trace map. Let $\chi: \mathbb{F}_{q}^{\times} \rightarrow \mathbb{C}^{\times}$be a multiplicative character. We extend $\chi$ to $\mathbb{F}_{q}$ by defining $\chi(0)=0$, including the trivial character $\chi_{0}$.

Definition 2.4. The Gauss sum corresponding to $\chi$ is

$$
\tau_{p}(\chi)=-\sum_{a \in \mathbb{F}_{q}} \chi(a) \lambda(a)=-\sum_{a \in \mathbb{F}_{q}} \chi(a) \zeta_{p}^{\operatorname{Tr}(a)}
$$

The Jacobi sum corresponding to $\chi_{1}$ and $\chi_{2}$ is

$$
J\left(\chi_{1}, \chi_{2}\right)=-\sum_{a \in \mathbb{F}_{q}} \chi_{1}(a) \chi_{2}(1-a)
$$

It can be shown that the Gauss sum and Jacobi sum satisfy the following properties:

1. $\tau_{p}\left(\chi_{0}\right)=1$ and $J\left(\chi_{0}, \chi_{0}\right)=2-q$.

2. $\tau_{p}(\bar{\chi})=\chi(-1) \overline{\tau_{p}(\chi)}$. 
3. If $\chi \neq \chi_{0}, \tau_{p}(\chi) \tau_{p}(\bar{\chi})=\chi(-1) q$. If $\chi_{1} \chi_{2} \neq \chi_{0}$, then $J\left(\chi_{1}, \chi_{2}\right)=\frac{\tau_{p}\left(\chi_{1}\right) \tau_{p}\left(\chi_{2}\right)}{\tau_{p}\left(\chi_{1} \chi_{2}\right)}$. Thus, if $\chi_{1}, \chi_{2}$ are characters of order dividing $m$, then $J\left(\chi_{1}, \chi_{2}\right)$ is an algebraic integer in $\mathbb{Q}\left(\zeta_{m}\right)$.

4. If $\chi \neq \chi_{0}$, then $\tau_{p}(\chi) \overline{\tau_{p}(\chi)}=q$. If $\chi_{1}, \chi_{2}, \chi_{1} \chi_{2} \neq \chi_{0}$, then $J\left(\chi_{1}, \chi_{2}\right) \overline{J\left(\chi_{1}, \chi_{2}\right)}=q$.

5. Let $\chi=\chi_{1} \cdots \chi_{n}$. If $\psi=\left.\chi\right|_{\mathbb{F}_{p}}=\psi_{0}$, the trivial character on $\mathbb{F}_{p}$, then

$$
\prod_{i=1}^{n} \tau_{p}\left(\chi_{i}\right)=(-1)^{n} \sum_{\operatorname{Tr}\left(a_{1}+\cdots+a_{n}\right)=0} \chi_{1}\left(a_{1}\right) \cdots \chi_{n}\left(a_{n}\right)+(-1)^{n-1} \sum_{\operatorname{Tr}\left(a_{1}+\cdots+a_{n}\right)=1} \chi_{1}\left(a_{1}\right) \cdots \chi_{n}\left(a_{n}\right) .
$$

If $\psi \neq \psi_{0}$, then

$$
\prod_{i=1}^{n} \tau_{p}\left(\chi_{i}\right)=(-1)^{n-1} \tau_{p}(\psi) \sum_{\operatorname{Tr}\left(a_{1}+\cdots+a_{n}\right)=1} \chi_{1}\left(a_{1}\right) \cdots \chi_{n}\left(a_{n}\right) .
$$

(In these sums, $\left(a_{1}, \ldots, a_{n}\right)$ ranges over all $n$-tuples of elements in $\mathbb{F}_{q}^{\times}$.)

Proof. The proof of properties 1 through 4 can be found in [14]. We prove property 5. Let $\mathbf{a}=\left(a_{1}, \cdots, a_{n}\right), z=z_{\mathbf{a}}=\operatorname{Tr}\left(a_{1}+\cdots+a_{n}\right)$ and $c_{i}=\frac{a_{i}}{z}$ if $z \neq 0$. Then 


$$
\begin{aligned}
\prod_{i=1}^{n} \tau_{p}\left(\chi_{i}\right) & =(-1)^{n} \sum_{\mathbf{a} \in\left(\mathbb{F}_{q}^{\times}\right)^{n}} \chi_{1}\left(a_{1}\right) \cdots \chi_{n}\left(a_{n}\right) \zeta_{p}^{z} \\
& =(-1)^{n}\left[\sum_{\left\{\mathbf{a} \in\left(\mathbb{F}_{q}^{\times}\right)^{n}: z=0\right\}} \chi_{1}\left(a_{1}\right) \cdots \chi_{n}\left(a_{n}\right)+\sum_{\left\{\mathbf{a} \in\left(\mathbb{F}_{q}^{\times}\right)^{n}: z \neq 0\right\}} \chi_{1}\left(a_{1}\right) \cdots \chi_{n}\left(a_{n}\right) \zeta_{p}^{z}\right] \\
& =(-1)^{n}\left[\sum_{\left\{\mathbf{a} \in\left(\mathbb{F}_{q}^{\times}\right)^{n}: z=0\right\}} \chi_{1}\left(a_{1}\right) \cdots \chi_{n}\left(a_{n}\right)+\sum_{\left\{\mathbf{a} \in\left(\mathbb{F}_{q}^{\times}\right)^{n}: z \neq 0\right\}} \chi_{1}\left(z c_{1}\right) \cdots \chi_{n}\left(z c_{n}\right) \zeta_{p}^{z}\right] \\
& =(-1)^{n}\left[\sum_{\left\{\mathbf{a} \in\left(\mathbb{F}_{q}^{\times}\right)^{n}: z=0\right\}} \chi_{1}\left(a_{1}\right) \cdots \chi_{n}\left(a_{n}\right)+\sum_{\left\{\mathbf{a} \in\left(\mathbb{F}_{q}^{\times}\right)^{n}: z \neq 0\right\}} \chi_{1}(z) \cdots \chi_{n}(z) \chi_{1}\left(c_{1}\right) \cdots \chi_{n}\left(c_{n}\right) \zeta_{p}^{z}\right] \\
& =(-1)^{n} \sum_{z=0} \chi_{1}\left(a_{1}\right) \cdots \chi_{n}\left(a_{n}\right)+(-1)^{n-1}\left(-\sum_{z \in \mathbb{F}_{p}^{\times}} \chi(z)_{p}^{z}\right)\left(\sum_{\operatorname{Tr}\left(c_{1}+\cdots+c_{n}\right)=1} \chi_{1}\left(c_{1}\right) \cdots \chi_{n}\left(c_{n}\right)\right) .
\end{aligned}
$$

The last equality follows because the set

$$
\left\{\left(z c_{1}, \ldots, z c_{n}\right): z \in \mathbb{F}_{p}^{\times},\left(c_{1}, \ldots, c_{n}\right) \in\left(\mathbb{F}_{q}^{\times}\right)^{n}, \operatorname{Tr}\left(c_{1}+\cdots+c_{n}\right)=1\right\}
$$

is equal to the set $\left\{\left(a_{1}, \ldots, a_{n}\right) \in\left(\mathbb{F}_{q}^{n}\right)^{\times}: z \neq 0\right\}$. If $\psi=\psi_{0}$, then $\tau_{p}(\psi)=-\sum_{z \in \mathbb{F}_{p}^{\times}} \chi(z) \zeta_{p}^{z}=$ 1 , otherwise there exists a $b \in \mathbb{F}_{p}^{\times}$such that $\psi(b) \neq 1$ so

$$
\operatorname{Tr}\left(b a_{1}+\cdots b a_{n}\right)=b \operatorname{Tr}\left(a_{1}+\cdots+a_{n}\right)
$$

and

$$
\begin{aligned}
\sum_{\operatorname{Tr}\left(a_{1}+\cdots+a_{n}\right)=0} \chi_{1}\left(a_{1}\right) \cdots \chi_{n}\left(a_{n}\right) & =\sum_{b \operatorname{Tr}\left(a_{1}+\cdots+a_{n}\right)=0} \chi_{1}\left(b a_{1}\right) \cdots \chi_{n}\left(b a_{n}\right) \\
& =\psi(b) \sum_{\operatorname{Tr}\left(a_{1}+\cdots+a_{n}\right)=0} \chi_{1}\left(a_{1}\right) \cdots \chi_{n}\left(a_{n}\right),
\end{aligned}
$$


which implies that $\sum_{z=0} \chi_{1}\left(a_{1}\right) \cdots \chi_{n}\left(a_{n}\right)=0$. Note that property 3 can be proved the same way by using $z=a_{1}+\cdots+a_{n}$ and $c_{i}=\frac{a_{i}}{z}$ if $z \neq 0$.

The Teichmüller character is defined as follows.

Definition 2.5. Let $q=p^{f}$. Let $\mathfrak{p}$ be one of the $\frac{\varphi(q-1)}{f}$ prime ideals of $\mathbb{Q}\left(\zeta_{q-1}\right)$ lying above the prime number $p$. Identify $\mathbb{Z}\left[\zeta_{q-1}\right](\bmod \mathfrak{p})$ with $\mathbb{F}_{q}$. The equation $X^{q-1}-1=0$ has distinct roots modulo $\mathfrak{p}$. Thus, there is a group isomorphism

$$
\omega: \mathbb{F}_{q}^{\times} \rightarrow \mu_{q-1}
$$

such that $\omega(a)=a(\bmod \mathfrak{p}) . \omega$ is a character of $\mathbb{F}_{q}^{\times}$, called the Teichmuller character corresponding to $\mathfrak{p}$.

The Teichmüller character generates the character group. Thus, any character $\chi$ of $\mathbb{F}_{q}^{\times}$ can be written as an integral power of $\omega$. Note that since $\omega$ has order $q-1$, the powers of $\omega$ can be expressed modulo $q-1$.

Now let $\mathfrak{P}$ be a prime above $\mathfrak{p}$ in $\mathbb{Q}\left(\zeta_{q-1}, \zeta_{p}\right)$. Let $k$ be an integer and first assume that $0 \leq k<q-1$. We may represent $k$ in its $p$-adic expansion by $k=k_{0}+k_{1} p+\cdots+$ $k_{f-1} p^{f-1}$, where $0 \leq k_{i}<p$. Define $s(k)$ and $\gamma(k)$ by $s(k)=k_{0}+k_{1}+\cdots k_{f-1}$ and $\gamma(k)=$ $k_{0} ! k_{1} ! \cdots k_{f-1} !$. For $k \geq q-1$, use $s\left(k^{\prime}\right)$ and $\gamma\left(k^{\prime}\right)$ where $k^{\prime} \equiv k(\bmod q-1)$ and $0 \leq k^{\prime}<$ $q-1$. We have 
Theorem 2.6 (Stickelberger). (See [9]) For any integer $k$,

$$
\frac{\tau_{p}\left(\omega^{-k}\right)}{\left(\zeta_{p}-1\right)^{s(k)}} \equiv \frac{1}{\gamma(k)} \quad(\bmod \mathfrak{P})
$$

where $\omega$ is the Teichmüller character, which generates the character group.

\subsection{Solutions of Norm Equations for $d=1,2,3$}

We begin by looking at three examples to motivate and introduce our study.

Example 2.1. Let $K_{1}=\mathbb{Q}\left(\zeta_{4}\right)$, where $\zeta_{4}$ is a primitive fourth root of unity. Let $p$ be a rational prime with $p \equiv 1(\bmod 4)$. p splits in $K_{1}$. That is, $p=d_{1} d_{2}$, where $N_{K_{1} / \mathbb{Q}}\left(d_{i}\right)=p$ for $i=1,2$. There exists $x+\zeta_{4} y \in \mathscr{O}_{K_{1}}=\mathbb{Z}\left[\zeta_{4}\right]$ such that $\left(x+\zeta_{4} y\right)=d_{i}$ because the class number of $K_{1}$ is equal to 1 . Therefore, $N_{K_{1} / \mathbb{Q}}\left(\left(x+\zeta_{4} y\right)\right)=\left(N_{K_{1} / \mathbb{Q}}\left(x+\zeta_{4} y\right)\right)=x^{2}+y^{2}=$ $N_{K_{1} / \mathbb{Q}}\left(d_{i}\right)=p$. Hence, any $p \equiv 1(\bmod 4)$ is representable as $x^{2}+y^{2}$, where $x, y \in \mathbb{Z}$.

We can actually represent these elements of norm $p$ in terms of Gauss sums. We recall that $\tau_{p}(\chi)=-\sum_{a \in \mathbb{Z} / p \mathbb{Z}} \chi(a) \zeta_{p}^{a}$ for the character $\chi$. Let $\omega$ be the Teichmüller character corresponding to a fixed prime ideal in $K_{1}$ above $p$. Define $\chi_{1}=\omega^{\frac{p-1}{4}}, \chi_{2}=$ $\omega^{\frac{p-1}{2}}$ and $\chi_{1} \chi_{2}=\omega^{\frac{3 p-3}{4}}$. From above, we see that $J\left(\chi_{1}, \chi_{2}\right)$ is an algebraic integer with $J\left(\chi_{1}, \chi_{2}\right) \overline{J\left(\chi_{1}, \chi_{2}\right)}=p$. Therefore, the element $x+\zeta_{4} y=\frac{\tau\left(\chi_{1}\right) \tau\left(\chi_{2}\right)}{\tau\left(\chi_{1} \chi_{2}\right)}$ in $\mathbb{Z}\left[\zeta_{4}\right]$ has norm $p$. We may further characterize the $x$ 's and y's by using Theorem 2.6. Since $\frac{p-1}{4}, \frac{p-2}{4}$ and $\frac{3 p-3}{4}$ are all less than $p-1$, they remain the same under $p$-adic expansion. We have, 
using the property $\tau_{p}(\chi) \tau_{p}(\bar{\chi})=\chi(-1) p$ and the Stickelberger Theorem (Theorem 2.6),

$$
\begin{aligned}
& x+\zeta_{4} y=\frac{\tau\left(\chi_{1}\right) \tau\left(\chi_{2}\right)}{\tau\left(\chi_{1} \chi_{2}\right)}=\frac{\tau\left(\omega^{\frac{p-1}{4}}\right) \tau\left(\omega^{\frac{p-1}{2}}\right)}{\tau\left(\omega^{\frac{3 p-3}{4}}\right)}=\frac{p \omega^{\frac{p-1}{4}}(-1)}{\tau\left(\omega^{-\frac{p-1}{4}}\right)} \cdot \frac{p \omega^{\frac{p-1}{2}}(-1)}{\tau\left(\omega^{-\frac{p-1}{2}}\right)} \cdot \frac{\tau\left(\omega^{-\frac{3 p-3}{4}}\right)}{p \omega^{\frac{3 p-3}{4}}(-1)} \\
& =\frac{p \tau\left(\omega^{-\frac{3 p-3}{4}}\right)}{\tau\left(\omega^{-\frac{p-1}{4}}\right) \tau\left(\omega^{-\frac{p-1}{2}}\right)} \equiv \frac{p \frac{\left(\zeta_{p}-1\right)^{\frac{3 p-3}{4}}}{\left(\frac{3 p-3}{4}\right) !}}{\frac{\left(\zeta_{p}-1\right)^{\frac{p-1}{4}}}{\left(\frac{p-1}{4}\right) !} \cdot \frac{\left(\zeta_{p}-1\right)^{\frac{p-1}{2}}}{\left(\frac{p-1}{2}\right) !}} \quad(\bmod \mathfrak{P}) \\
& =\frac{p\left(\frac{p-1}{4}\right) !\left(\frac{p-1}{2}\right) !}{\left(\frac{3 p-3}{4}\right) !} \quad(\bmod \mathfrak{P}), \\
& x+\zeta_{4}^{-1} y=\frac{\overline{\tau\left(\chi_{1}\right) \tau\left(\chi_{2}\right)}}{\overline{\tau\left(\chi_{1} \chi_{2}\right)}}=\frac{\tau\left(\overline{\chi_{1}}\right)}{\chi_{1}(-1)} \cdot \frac{\tau\left(\overline{\chi_{2}}\right)}{\chi_{2}(-1)} \cdot \frac{\chi_{1} \chi_{2}(-1)}{\tau\left(\overline{\chi_{1} \chi_{2}}\right)}=\frac{\tau\left(\overline{\chi_{1}}\right) \tau\left(\overline{\chi_{2}}\right)}{\tau\left(\overline{\chi_{1} \chi_{2}}\right)} \\
& =\frac{\tau\left(\omega^{-\frac{p-1}{4}}\right) \tau\left(\omega^{-\frac{p-1}{2}}\right)}{\tau\left(\omega^{-\frac{3 p-3}{4}}\right)} \\
& \equiv \frac{\left(\frac{3 p-3}{4}\right) !}{\left(\frac{p-1}{4}\right) !\left(\frac{p-1}{2}\right) !} \quad(\bmod \mathfrak{P}) \text {. }
\end{aligned}
$$

Hence,

$$
2 x \equiv \frac{\left(\frac{3 p-3}{4}\right) !}{\left(\frac{p-1}{4}\right) !\left(\frac{p-1}{2}\right) !} \quad(\bmod p)
$$

or

$$
\begin{aligned}
x & \equiv \frac{\left(\frac{3 p-3}{4}\right) !}{2 \cdot\left(\frac{p-1}{4}\right) !\left(\frac{p-1}{2}\right) !} \quad(\bmod p) \\
& =\frac{1}{2}\left(\begin{array}{c}
\frac{3 p-3}{4} \\
\frac{p-1}{4}
\end{array}\right) \quad(\bmod p),
\end{aligned}
$$

where $\left(\begin{array}{l}n \\ r\end{array}\right)=\frac{n !}{r !(n-r) !}$ is a binomial coefficient. 
We can also get the congruence for y by subtracting $x+\zeta_{4}^{-1} y$ from $x+\zeta_{4} y$ :

$$
2 \zeta_{4} y \equiv \frac{\left(\frac{3 p-3}{4}\right) !}{\left(\frac{p-1}{4}\right) !\left(\frac{p-1}{2}\right) !} \quad(\bmod \mathfrak{P})
$$

Wilson's theorem states that $(p-1) ! \equiv-1(\bmod p)$. That is,

$$
\begin{aligned}
(p-1) ! & =(p-1)(p-2) \cdots\left(p-\frac{p-1}{2}\right)\left(\frac{p-1}{2}\right) ! \\
& =(-1)^{\frac{p-1}{2}}\left(\frac{p-1}{2}\right) !^{2} \quad(\bmod p) \\
& \equiv-1 \quad(\bmod p)
\end{aligned}
$$

or

$$
\left(\frac{p-1}{2}\right) !^{2} \equiv(-1)^{\frac{3-p}{2}} \quad(\bmod p) .
$$

Since $p \equiv 1(\bmod 4)$, we see that $\left(\frac{p-1}{2}\right) !^{2} \equiv-1(\bmod p)$. Thus, we can choose $\mathfrak{P}$ such that $\zeta_{4} \equiv\left(\frac{p-1}{2}\right) !(\bmod \mathfrak{P})$. Finally,

$$
y \equiv \frac{\left(\frac{3 p-3}{4}\right) !}{2\left(\frac{p-1}{4}\right) !\left(\frac{p-1}{2}\right) !^{2}} \equiv-\frac{\left(\frac{3 p-3}{4}\right) !}{2\left(\frac{p-1}{4}\right) !} \quad(\bmod p)
$$

Now, $N_{K_{1} / \mathbb{Q}}\left(\left(x+\zeta_{4} y\right)\right)=x^{2}+y^{2}=$ p implies that $x^{2}<p$ and $y^{2}<p$ so that $x<\sqrt{p}<\frac{p}{2}$ and $y<\sqrt{p}<\frac{p}{2}$. Note that $\sqrt{p}<\frac{p}{2}$ is true for $p \geq 5$.

Definition 2.7. Let $\alpha=\frac{a}{b} \in \mathbb{Q}, a, b \in \mathbb{Z},(n, b)=1$ where $n$ is odd. We define the notation $\langle\alpha\rangle$ to be the integer such that $\langle\alpha\rangle \equiv \alpha(\bmod n)$ and $-\frac{n-1}{2} \leq\langle\alpha\rangle \leq \frac{n-1}{2}$. 
Remark 1. Note that $\langle-\alpha\rangle=-\langle\alpha\rangle$. To see this, we have $\langle-\alpha\rangle \equiv-\alpha(\bmod n)$ and $-\langle\alpha\rangle \equiv-\alpha(\bmod n)$. Also, $\frac{n-1}{2} \geq-\langle\alpha\rangle \geq-\frac{n-1}{2}$. Thus, $\langle-\alpha\rangle=-\langle\alpha\rangle$.

Lemma 2.8. Let $n>0$ be a natural number such that $n \equiv 1(\bmod 4)$. Then $n$ is prime if and only if

$$
\left(\frac{n-1}{2}\right) !^{2} \equiv-1 \quad(\bmod n)
$$

Furthermore, when $n$ is composite, $n \neq 9$,

$$
\left(\frac{n-1}{2}\right) ! \equiv 0 \quad(\bmod n)
$$

Proof. We showed above that if $n$ is a prime such that $n \equiv 1(\bmod 4)$, then $n$ satisfies (2.1). Suppose now that $n$ is composite and $n \equiv 1(\bmod 4)$. Let $p$ be a prime such that $p \mid n$. Then $p<\frac{n-1}{2}$ and $\frac{n}{p}<\frac{n-1}{2}$. If $n$ is not the square of a prime, then $p$ and $\frac{n}{p}$ are distinct and therefore $\left(\frac{n-1}{2}\right) ! \equiv 0(\bmod n)$. If $n=p^{2}$ for some prime $p$, as long as $p \neq 3$, then $2 p<\frac{n-1}{2}$ so that $p^{2} \mid\left(\frac{n-1}{2}\right) !$ and $\left(\frac{n-1}{2}\right) ! \equiv 0(\bmod n)$ in this case as well. If $n=9$, then $\left(\frac{9-1}{2}\right) !=24 \equiv 0(\bmod 3)$.

Theorem 2.9. Let $n>0$ be a natural number, not a square, such that $n \equiv 1(\bmod 4)$. The pair

$$
\left(\left\langle\frac{1}{2}\left(\begin{array}{c}
\frac{3 n-3}{4} \\
\frac{n-1}{4}
\end{array}\right)\right\rangle,\left\langle\frac{\left(\frac{3 n-3}{4}\right) !}{2\left(\frac{n-1}{4}\right) !}\right\rangle\right)
$$

is a solution to $x^{2}+y^{2}=n$ if and only if $n$ is prime. 
Proof. We saw above that if $p \equiv 1(\bmod 4)$ and $p$ is prime, then $(2.3)$ is a solution to $x^{2}+y^{2}=p$

Suppose now that $n$ is a composite number, not a square and $n \equiv 1(\bmod 4)$. Suppose further that

$$
\left\langle\frac{1}{2}\left(\begin{array}{c}
\frac{3 n-3}{4} \\
\frac{n-1}{4}
\end{array}\right)\right\rangle^{2}+\left\langle\frac{\left(\frac{3 n-3}{4}\right) !}{2\left(\frac{n-1}{4}\right) !}\right\rangle^{2}=n .
$$

It follows from congruence (2.2) and direct computations for $n=9$ that

$$
\frac{\left(\frac{3 n-3}{4}\right) !}{2\left(\frac{n-1}{4}\right) !}=\frac{1}{2}\left(\begin{array}{c}
\frac{3 n-3}{4} \\
\frac{n-1}{4}
\end{array}\right)\left(\frac{n-1}{2}\right) ! \equiv 0 \quad(\bmod n)
$$

Equation (2.4) implies

$$
\left\langle\frac{1}{2}\left(\begin{array}{c}
\frac{3 n-3}{4} \\
\frac{n-1}{4}
\end{array}\right)\right\rangle^{2}=n
$$

contradicting the condition that $n$ is not a square.

Example 2.2. Now consider $K_{2}=\mathbb{Q}\left(\zeta_{6}\right)=\mathbb{Q}\left(\zeta_{3}\right)=\mathbb{Q}(\sqrt{-3})$ and let $p$ be a rational prime with $p \equiv 1(\bmod 3)$. $p$ splits in $K_{2}$, so $p=d_{1} d_{2}$ with $N_{K_{2} / \mathbb{Q}}\left(d_{i}\right)=p$ for $i=1,2$. Again, there exists $x+\zeta_{6} y \in \mathscr{O}_{K_{2}}=\mathbb{Z}\left[\frac{1+\sqrt{-3}}{2}\right]=\mathbb{Z}\left[\zeta_{6}\right]$ such that $\left(x+\zeta_{6} y\right)=d_{1}$. Therefore, $N_{K_{2} / \mathbb{Q}}\left(\left(x+\zeta_{6} y\right)\right)=\left(x+\zeta_{6} y\right)\left(x+\zeta_{6}^{-1} y\right)=x^{2}+x y+y^{2}=\left(x+\frac{y}{2}\right)^{2}+\frac{3 y^{2}}{4}=p$.

Similar to the case $K_{1}=\mathbb{Q}\left(\zeta_{4}\right)$, we define $\chi_{1}=\omega^{\frac{p-1}{6}}, \chi_{2}=\omega^{\frac{p-1}{3}}$, hence $\chi_{1} \chi_{2}=\omega^{\frac{3 p-3}{6}}$ 
and we have

$$
\begin{aligned}
x+\zeta_{6} y & =\frac{\tau_{p}\left(\chi_{1}\right) \tau_{p}\left(\chi_{2}\right)}{\tau_{p}\left(\chi_{1} \chi_{2}\right)}=\frac{\tau_{p}\left(\omega^{\frac{p-1}{6}}\right) \tau\left(\omega^{\frac{p-1}{3}}\right)}{\tau_{p}\left(\omega^{\frac{3 p-3}{6}}\right)}=\frac{p \tau_{p}\left(\omega^{-\frac{3 p-3}{6}}\right)}{\tau_{p}\left(\omega^{-\frac{p-1}{6}}\right) \tau_{p}\left(\omega^{-\frac{p-1}{3}}\right)} \\
& \equiv \frac{p\left(\frac{p-1}{6}\right) !\left(\frac{p-1}{3}\right) !}{\left(\frac{3 p-3}{6}\right) !}(\bmod \mathfrak{P}), \\
x+\zeta_{6}^{-1} y & \equiv \frac{\left(\frac{3 p-3}{6}\right) !}{\left(\frac{p-1}{6}\right) !\left(\frac{p-1}{3}\right) !} \quad(\bmod \mathfrak{P}) .
\end{aligned}
$$

Adding the two equations,

$$
\begin{aligned}
2 x+y & \equiv \frac{\left(\frac{3 p-3}{6}\right) !}{\left(\frac{p-1}{6}\right) !\left(\frac{p-1}{3}\right) !} \quad(\bmod p) \\
& =\left(\begin{array}{c}
\frac{3 p-3}{6} \\
\frac{p-1}{6}
\end{array}\right) \quad(\bmod p)
\end{aligned}
$$

Subtracting the two equations,

$$
\sqrt{-3} y \equiv\left(\begin{array}{c}
\frac{3 p-3}{6} \\
\frac{p-1}{6}
\end{array}\right) \quad(\bmod p)
$$

One may note that square roots modulo p can be computed. We will proceed without this fact. From $(2 x+y)^{2}=4 p-3 y^{2}$, we see that $(2 x+y)^{2}<4$ so that $|2 x+y|<2 \sqrt{p}<\frac{p}{2}$ if $4 \sqrt{p}<p$, or $16<p$. That is, if $p \geq 17$, then $|2 x+y|<\frac{p}{2}$. Let $\alpha=\left(\begin{array}{c}\frac{3 p-3}{6} \\ \frac{p-1}{6}\end{array}\right)$ and $\langle\alpha\rangle \equiv \alpha$ $(\bmod p)$ be the integer such that $-\frac{p-1}{2} \leq\langle\alpha\rangle \leq \frac{p-1}{2}$. In this case, $2 x+y=\langle\alpha\rangle$ and $y^{2}=\frac{4 p-(2 x+y)^{2}}{3}$ implies

$$
y= \pm \sqrt{\frac{4 p-\langle\alpha\rangle^{2}}{3}}
$$


and

$$
x=\frac{\langle\alpha\rangle-y}{2}
$$

Theorem 2.10. Let $p \equiv 1(\bmod 3), p \geq 19$. Let $\alpha=\left(\begin{array}{c}\frac{3 p-3}{6} \\ \frac{p-1}{6}\end{array}\right)$. Then

$$
\left(\frac{\langle\alpha\rangle-\sqrt{\frac{4 p-\langle\alpha\rangle^{2}}{3}}}{2}, \sqrt{\frac{4 p-\langle\alpha\rangle^{2}}{3}}\right)
$$

is a solution to $\left(x+\frac{y}{2}\right)^{2}+\frac{3 y^{2}}{4}=p$.

Remark 2. Theorem 2.10 holds for $p=13$ as well. This can be verified directly.

Example 2.3. Let $K_{3}=\mathbb{Q}(\sqrt{-2})$. Then $K_{3} \subset L_{3}=\mathbb{Q}\left(\zeta_{8}\right)$. Let $p$ be a rational prime. If $p \equiv 1(\bmod 8)$ or $p \equiv 3(\bmod 8)$, then $p$ plits in $K_{3}$. Say $p \mathscr{O}_{K_{3}}=d_{1} d_{2}$. Let $x+\sqrt{-2} y$ and $x-\sqrt{-2} y \in \mathscr{O}_{K_{3}}$ such that $(x+\sqrt{-2} y)=d_{1}$ and $(x-\sqrt{-2} y)=d_{2}$. We have $N_{K_{3} / \mathbb{Q}}(x+$ $\sqrt{-2} y)=x^{2}+2 y^{2}=N_{K_{3} / \mathbb{Q}}\left(d_{1}\right)=p$.

$\operatorname{Gal}\left(L_{3} / \mathbb{Q}\right) \cong \mathbb{Z} / 2 \mathbb{Z} \times \mathbb{Z} / 2 \mathbb{Z}$ and Gal $\left(L_{3} / K_{3}\right)$ is a subgroup of order 2 . Define $\sigma_{i} \in$ $\operatorname{Gal}\left(L_{3} / \mathbb{Q}\right)$ by $\sigma_{i}\left(\zeta_{8}\right)=\zeta_{8}^{i}$. Now, $\sqrt{-2}=\zeta_{8}+\zeta_{8}^{3}$ so $\sigma_{1}(\sqrt{-2})=\sqrt{-2}$ and $\sigma_{3}(\sqrt{-2})=$ $\sqrt{-2}$. Therefore, Gal $\left(L_{3} / K_{3}\right)=\left\{\sigma_{1}, \sigma_{3}\right\}$.

If $p \equiv 1(\bmod 8)$, let $\chi_{1}=\omega^{\frac{p-1}{8}}, \chi_{2}=\omega^{\frac{3 p-3}{8}}$. Note that $J\left(\chi_{1}, \chi_{2}\right)=$ $\frac{\tau_{p}\left(\omega^{\frac{p-1}{8}}\right) \tau_{p}\left(\omega^{\frac{3 p-3}{8}}\right)}{\tau_{p}\left(\omega^{\frac{p-1}{2}}\right)} \in K_{3}$ since Gal $\left(L_{3} / K_{3}\right)$ fixes this Jacobi sum. Recall that $N_{K_{3} / \mathbb{Q}}\left(J\left(\chi_{1}, \chi_{2}\right)\right)=p$ since $\tau_{p}(\chi) \overline{\tau_{p}(\chi)}=p$. As above, $\frac{p-1}{8}, \frac{3 p-3}{8}$ and $\frac{p-1}{2}$ are less than $p-1$, so they remain the same under $p$-adic expansion. We have, using Stickel- 
berger's Theorem,

$$
\begin{aligned}
x+\sqrt{-2} y & =\frac{\tau_{p}\left(\omega^{\frac{p-1}{8}}\right) \tau_{p}\left(\omega^{\frac{3 p-3}{8}}\right)}{\tau_{p}\left(\omega^{\frac{p-1}{2}}\right)}=\frac{p \tau_{p}\left(\omega^{-\frac{p-1}{2}}\right)}{\tau_{p}\left(\omega^{-\frac{p-1}{8}}\right) \tau_{p}\left(\omega^{-\frac{3 p-3}{8}}\right)} \equiv \frac{p\left(\frac{p-1}{8}\right) !\left(\frac{3 p-3}{8}\right) !}{\left(\frac{p-1}{2}\right) !} \\
& \equiv 0 \quad(\bmod \mathfrak{P}), \\
x-\sqrt{-2} y & =\frac{\tau_{p}\left(\omega^{-\frac{p-1}{8}}\right) \tau_{p}\left(\omega^{-\frac{3 p-3}{8}}\right)}{\tau_{p}\left(\omega^{-\frac{4 p-4}{8}}\right)} \equiv \frac{\left(\frac{p-1}{2}\right) !}{\left(\frac{p-1}{8}\right) !\left(\frac{3 p-3}{8}\right) !} \quad(\bmod \mathfrak{P})
\end{aligned}
$$

so that

$$
x \equiv \frac{1}{2} \frac{\left(\frac{p-1}{2}\right) !}{\left(\frac{p-1}{8}\right) !\left(\frac{3 p-3}{8}\right) !} \quad(\bmod p) .
$$

Hence,

$$
x=\left\langle\frac{1}{2}\left(\begin{array}{c}
\frac{p-1}{2} \\
\frac{p-1}{8}
\end{array}\right)\right\rangle
$$

because $x^{2}+2 y^{2}=p$ implies $|x|<\frac{p}{2}$.

If $p \equiv 3(\bmod 8)$, then $q=p^{2} \equiv 1(\bmod 8)$. Let $\chi=\omega^{\frac{p^{2}-1}{8}}$, and $\psi=\left.\chi\right|_{\mathbb{F}_{p}}$. Note that $\psi(c)=c^{\frac{p-1}{2}\left(\frac{p+1}{4}\right)}=c^{\frac{p-1}{2}}(\bmod \mathfrak{P})$ because $\frac{p+1}{4}$ is odd. Let

$$
\begin{aligned}
& Z=\frac{\tau_{p}(\chi)}{\tau_{p}(\psi)}=\sum_{c \in \mathbb{F}_{q}} \chi(c), \\
& \operatorname{Tr}(c)=1
\end{aligned}
$$

according to property 5 on page 10. It is clear that $Z$ is invariant under $\sigma_{p}=\sigma_{3}$, so $Z \in \mathscr{O}_{K_{3}}$ and $N_{K_{3} / \mathbb{Q}}(Z)=\frac{q}{p}=p$. Therefore, $x+\sqrt{-2} y=Z$ works in this case. 
We have

$$
\begin{aligned}
\frac{p^{2}-1}{8} & =\frac{3 p-1}{8}+\frac{p-3}{8} p, \\
x+\sqrt{-2} y & =\frac{p \tau_{p}\left(\psi^{-1}\right)}{\tau_{p}\left(\chi^{-1}\right)} \equiv 0 \quad(\bmod \mathfrak{P}), \\
x-\sqrt{-2} y & =\frac{\tau_{p}\left(\chi^{-1}\right)}{\tau_{p}\left(\psi^{-1}\right)} \equiv \frac{\left(\frac{p-1}{2}\right) !}{\left(\frac{p-3}{8}\right) !\left(\frac{3 p-1}{8}\right) !}=\left(\begin{array}{c}
\frac{p-1}{2} \\
\frac{p-3}{8}
\end{array}\right) \quad(\bmod \mathfrak{P})
\end{aligned}
$$

because $\frac{\tau_{p}\left(\chi^{-1}\right)}{\left(\zeta_{p}-1\right)^{\frac{p-1}{2}}} \equiv \frac{1}{\left(\frac{3 p-1}{8}\right) !\left(\frac{p-3}{8}\right) !}(\bmod \mathfrak{P})$ and $\frac{\tau_{p}\left(\psi^{-1}\right)}{\left(\zeta_{p}-1\right)^{\frac{p-1}{2}}} \equiv \frac{1}{\left(\frac{p-1}{2}\right) !}(\bmod \mathfrak{P})$ by Stickelberger's Theorem (Theorem 2.6). Thus,

$$
x \equiv \frac{1}{2}\left(\begin{array}{c}
\frac{p-1}{2} \\
\frac{p-3}{8}
\end{array}\right) \quad(\bmod p)
$$

and

$$
x=\left\langle\frac{1}{2}\left(\begin{array}{c}
\frac{p-1}{2} \\
\frac{p-3}{8}
\end{array}\right)\right\rangle
$$

because $|x|<\frac{p}{2}$.

Theorem 2.11. Let $\alpha=\left\{\begin{array}{ll}\frac{1}{2}\left(\begin{array}{l}\frac{p-1}{2} \\ \left.\frac{p-1}{8}\right)\end{array}\right. & \text { if } p \equiv 1(\bmod 8) \\ \frac{1}{2}\left(\begin{array}{l}\frac{p-1}{2} \\ \left.\frac{p-3}{8}\right)\end{array}\right. & \text { if } p \equiv 3(\bmod 8)\end{array}\right.$. Then

$$
\left(\langle\alpha\rangle, \sqrt{\frac{p-\langle\alpha\rangle^{2}}{2}}\right)
$$

is a solution to $x^{2}+2 y^{2}=p$.

We wish to generalize these results to all imaginary quadratic extensions of $\mathbb{Q}$. 


\section{Chapter 3}

\section{The General Case}

Throughout, unless stated otherwise, we will let $q=p^{f}$, where $p$ is prime.

\subsection{The Gross-Koblitz Formula}

Before we get to the Gross-Koblitz theorem, we need to the following:

Definition 3.1. The $p$-adic gamma function is $\Gamma_{p}(k)=(-1)^{k} \prod_{j<k, p \downarrow j} j$, for $k=1,2,3, \ldots$ (When $k=1$, we define $\left.\Gamma_{p}(1)=-1.\right)$

Lemma 3.2. Let $s \in \mathbb{N}$ such that $s \equiv s_{0}\left(\bmod p^{n}\right)$. Let $n \neq 2$ if $p=2$. Then $\Gamma_{p}(s)$ $\left(\bmod p^{n}\right) \equiv \Gamma_{p}\left(s_{0}\right)$

Proof. We will follow the proof in [8], page 90. Let $s$ and $s_{0}$ be natural numbers, $s>s_{0}$. We will show that if $s \equiv s_{0}\left(\bmod p^{n}\right), n \neq 2$ if $p=2$, then $\Gamma_{p}(s)\left(\bmod p^{n}\right) \equiv \Gamma_{p}\left(s_{0}\right)$. To see this, write $s=s_{0}+k p^{n}$. We will prove this for $k=1$ first. That is, assume $s=s_{0}+p^{n}$. 
$\Gamma_{p}(s)\left(\bmod p^{n}\right) \equiv \Gamma_{p}\left(s_{0}\right)$ is true if and only if

$$
1 \equiv \frac{\Gamma_{p}(s)}{\Gamma_{p}\left(s_{0}\right)}=(-1)^{s-s_{0}} \prod_{\substack{s_{0} \leq j<s \\ p \nmid X_{j}}} j \quad\left(\bmod p^{n}\right)=(-1)^{p} \prod_{\substack{s_{0} \leq j<s \\ p \not j}} j \quad\left(\bmod p^{n}\right)
$$

Now since $s=s_{0}+p^{n}$, this product runs through every congruence class in $\left(\frac{\mathbb{Z}}{p^{n}} \mathbb{Z}\right)^{\times}$ exactly once and we have

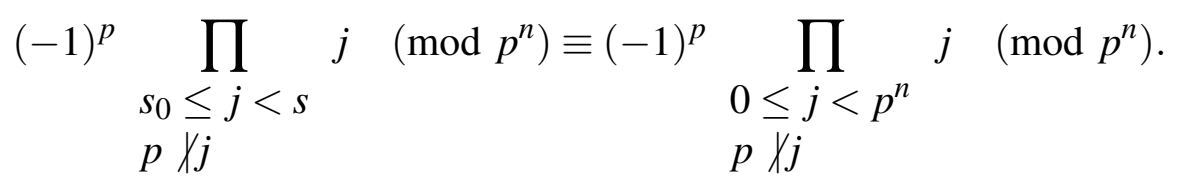

Therefore, to complete the case $k=1$, we need

$$
\prod_{\substack{0 \leq j<p^{n} \\ p \times j}} j \equiv(-1)^{p} \quad\left(\bmod p^{n}\right)
$$

In the product, we can pair a number with its inverse. That is, there exists $j^{-1} \in\left(\mathbb{Z} / p^{n} \mathbb{Z}\right)^{\times}$ such that $j j^{-1}=1$. Therefore, the product simplifies to

$$
\begin{aligned}
& \prod_{\substack{0 \leq j<p^{n} \\
p \times j}} j \equiv \underset{\substack{1 \leq j<p^{n} \\
j^{2} \equiv 1 \quad\left(\bmod p^{n}\right)}}{ } j . \\
& p \times j
\end{aligned}
$$

Suppose $j^{2} \equiv 1\left(\bmod p^{n}\right)$. Then $j=p a \pm 1$, where $a$ is even if $p=2, n \geq 3$. Then $j^{2}=1 \pm 2 p a+p^{2} a^{2} \equiv 1\left(\bmod p^{n}\right) \Leftrightarrow 2 p a \equiv 0\left(\bmod p^{n}\right)$. So $j=1$ or $p^{n}-1$ if $p$ is odd; 
$j=1$ if $p=2$ and $n=1 ; j=1,2^{n}-1,2^{n-1}+1$ or $2^{n-1}-1$ if $p=2, n \geq 3$. In all cases,

$$
\begin{aligned}
& \prod_{\substack{1 \leq j<p^{n} \\
j^{2} \equiv 1 \quad\left(\bmod p^{n}\right) \\
p \nmid j}} j \equiv(-1)^{*} \quad\left(\bmod p^{n}\right) . \\
&
\end{aligned}
$$

We have proven the congruence for $k=1$. To show this is true for $s=s_{0}+k p^{n}, k \neq 1$ we need

$$
1 \equiv \frac{\Gamma_{p}(s)}{\Gamma_{p}\left(s_{0}\right)}=(-1)^{k p^{n}} \prod_{\substack{s_{0} \leq j<s_{0}+k p^{n} \\ p \not j}} j\left(\bmod p^{n}\right)
$$

This is obtained by multiplying together the following congruences:

$$
\begin{aligned}
& 1 \equiv \frac{\Gamma_{p}\left(s_{0}+p^{n}\right)}{\Gamma_{p}\left(s_{0}\right)}=(-1)^{s_{0}+p^{n}-s_{0}} \prod_{\substack{s_{0} \leq j<s_{0}+p^{n} \\
p \nmid j}} j \\
& 1 \equiv \frac{\Gamma_{p}\left(s_{0}+2 p^{n}\right)}{\Gamma_{p}\left(s_{0}+p^{n}\right)}=(-1)^{s_{0}+2 p^{n}-\left(s_{0}+p^{n}\right)} \prod_{\substack{s_{0}+p^{n} \leq j<s_{0}+2 p^{n} \\
p \nmid j}} j \\
& \vdots \\
& 1 \equiv \frac{\Gamma_{p}\left(s_{0}+k p^{n}\right)}{\Gamma_{p}\left(s_{0}+(k-1) p^{n}\right)}=(-1)^{s_{0}+k p^{n}-\left(s_{0}+(k-1) p^{n}\right)} \prod_{\substack{s_{0}+(k-1) p^{n} \leq j<s_{0}+k p^{n} \\
p \nmid j}} j .
\end{aligned}
$$

Proposition 3.3. $\Gamma_{p}$ extends to a well-defined continuous function $\Gamma_{p}: \mathbb{Z}_{p} \rightarrow \mathbb{Z}_{p}^{\times}$defined 
by

$$
\Gamma_{p}(a)=\lim _{t \rightarrow a, t \in \mathbb{N}}(-1)^{t} \prod_{j<t, p \downarrow j} j
$$

where $\{t\}$ is any sequence of natural numbers converging to $a \in \mathbb{Z}_{p}$.

Proof. Take a sequence $\left\{t_{i}\right\}$ approaching $a$. Then the sequence $\left\{\Gamma_{p}\left(t_{i}\right)\right\}$ converges. Define $\Gamma_{p}(a)=\lim _{i \rightarrow \infty}(-1)^{t_{i}} \prod_{j<t_{i}, p \backslash j} j$.

To show that $\Gamma_{p}(a)$ is well-defined, suppose $\left\{s_{i}\right\}$ and $\left\{s_{i}^{\prime}\right\}$ are two Cauchy sequences of natural numbers converging to $a$. Then $\left\{s_{i}\right\}$ and $\left\{s_{i}^{\prime}\right\}$ are in the same equivalence class of Cauchy sequences, so $\left|s_{i}-s_{i}^{\prime}\right|_{p} \rightarrow 0$ as $i \rightarrow \infty$. We need to show that this implies $\left|\Gamma_{p}\left(s_{i}\right)-\Gamma_{p}\left(s_{i}^{\prime}\right)\right|_{p} \rightarrow 0$ as $i \rightarrow \infty$. This is clear: as shown in Lemma 3.2, if $s_{i} \equiv s_{i}^{\prime}\left(\bmod p^{n}\right)$ then $\Gamma_{p}\left(s_{i}\right) \equiv \Gamma_{p}\left(s_{i}^{\prime}\right)\left(\bmod p^{n}\right)$.

Lemma 3.4. Let $s=s_{0}+s_{1} p+s_{2} p^{2}+\cdots \in \mathbb{Z}_{p}$ such that $s_{i} \in \mathbb{Z}, 0 \leq s_{i}<p$. Let $\left\{k_{i}\right\}=$ $\left\{s_{0}, s_{0}+s_{1} p, s_{0}+s_{1} p+s_{2} p^{2}, \ldots\right\}$ for $i=0,1,2, \ldots$ be the sequence of partial sums of $s$, $k_{n-1} \equiv s\left(\bmod p^{n}\right)$ for $n=1,2,3, \ldots(n \neq 2$ if $p=2)$. Then $\Gamma_{p}\left(k_{n-1}\right) \equiv \Gamma_{p}(s)\left(\bmod p^{n}\right)$. Furthermore, if $s, s^{\prime} \in \mathbb{Z}_{p}$ and $s \equiv s^{\prime}\left(\bmod p^{n}\right)$, then $\Gamma_{p}(s) \equiv \Gamma_{p}\left(s^{\prime}\right)\left(\bmod p^{n}\right)$.

Proof. It is clear that $\left\{k_{i}\right\}$ converges to $s$ and $\left\{\Gamma_{p}\left(k_{i}\right)\right\}$ converges to $\Gamma_{p}(s)$. Also, $\Gamma_{p}\left(k_{i}\right) \equiv$ $\Gamma_{p}\left(k_{i+1}\right)\left(\bmod p^{i+1}\right)$ so $\Gamma_{p}\left(k_{n-1}\right) \equiv \Gamma_{p}\left(k_{i}\right)\left(\bmod p^{n}\right)$ for $i=n, n+1, \ldots$ Since $\Gamma_{p}(s)=$ $\lim _{i \rightarrow \infty} \Gamma_{p}\left(k_{i}\right)$, this implies that $\Gamma_{p}(s)-\Gamma_{p}\left(k_{i}\right) \equiv 0\left(\bmod p^{n}\right)$ for a large enough $i$. Therefore, $\Gamma_{p}(s) \equiv \Gamma_{p}\left(k_{i}\right)\left(\bmod p^{n}\right)$ so that $\Gamma_{p}(s) \equiv \Gamma_{p}\left(k_{n-1}\right)\left(\bmod p^{n}\right)$. 
Now if $s, s^{\prime} \in \mathbb{Z}_{p}$ and $s \equiv s^{\prime}\left(\bmod p^{n}\right)$, then $k_{n-1}=k_{n-1}^{\prime}, \Gamma_{p}(s) \equiv \Gamma_{p}\left(k_{n-1}\right)\left(\bmod p^{n}\right)$ and $\Gamma_{p}\left(s^{\prime}\right) \equiv \Gamma_{p}\left(k_{n-1}^{\prime}\right)\left(\bmod p^{n}\right)$. Also, $\Gamma_{p}\left(k_{n-1}\right)=\Gamma_{p}\left(k_{n-1}^{\prime}\right)$ so $\Gamma_{p}(s) \equiv \Gamma_{p}\left(s^{\prime}\right)\left(\bmod p^{n}\right)$

It can be shown that $\Gamma_{p}$ satisfies the following properties:

1. $\frac{\Gamma_{p}(s+1)}{\Gamma_{p}(s)}=\left\{\begin{array}{ll}-s & \text { if } s \in \mathbb{Z}_{p}^{\times} \\ -1 & \text { if } s \in p \mathbb{Z}_{p}\end{array}\right.$.

2. Suppose $p$ is odd. If $s \in \mathbb{Z}_{p}$, write $s=s_{0}+p s_{1}$, where $s_{0} \in\{1,2, \ldots, p\}$ is the first digit in $s$ unless $s \in p \mathbb{Z}_{p}, s_{0}=p$. Then $\Gamma_{p}(s) \Gamma_{p}(1-s)=(-1)^{s_{0}}$.

3. If $p=2$, then $\Gamma_{p}(s) \Gamma_{p}(1-s)=\left\{\begin{array}{ll}-1 & \text { if } s \equiv 0,1(\bmod 4) \\ 1 & \text { if } s \equiv 2,3(\bmod 4)\end{array}\right.$.

See [8] for the proof.

We are now ready to state the Gross-Koblitz theorem:

Theorem 3.5 (Gross-Koblitz). (See [2]) Let $f \geq 1$ and $q=p^{f}$. Let $\pi \in \mathscr{K}=\mathbb{Q}_{p}\left(\zeta_{p}\right)$ be such that $\pi^{p-1}=-p$. Let $\mathscr{L}=\mathscr{K}\left(\zeta_{q-1}\right)$ and $\zeta_{\pi}$ be the unique $p$-th root of unity congruent to $1+\pi$ modulo $\mathfrak{p}^{2}$, where $\mathfrak{p}$ is the prime ideal above $p$ in $\mathbb{Q}\left(\zeta_{p}\right)$. For $0 \leq a<$ $q-1$, we have

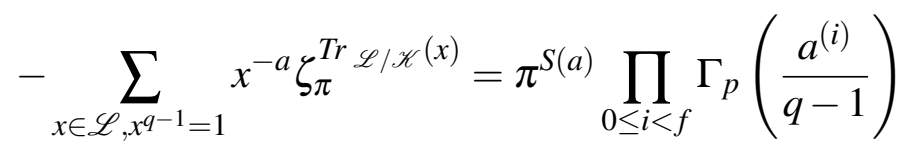

where $S(a)$ is the sum of the digits of a in base $p$, and the integers $0 \leq a^{(i)}<q-1$ have p-adic expansions obtained by cyclic permutation from that of a. 
Note that for $a \in \mathbb{Z}_{p}$, it makes sense to raise $\zeta_{\pi}$ to the $a$ power, since $\zeta_{\pi}$ is a $p$-th root of unity.

Remark 3. When $f=1$, we have $\mathscr{K}=\mathscr{L}$, since $\mathbb{Q}_{p}$ contains the $(p-1)$ th roots of unity.

The Gaussian sum in Theorem 3.5 becomes

$$
-\sum_{x \in \mathscr{K}, x^{p-1}=1} x^{-a} \zeta_{\pi}^{x}=-\sum_{x \in \mu_{p-1}} x^{-a} \zeta_{\pi}^{x}
$$

Now let $\omega$ be the Teichmüller character corresponding to $\mathfrak{p}$, defined by Definition 2.5 . Let $r_{x} \in \mathbb{F}_{p}^{\times}$be such that $\omega\left(r_{x}\right)=x \in \mu_{p-1}$. We can then rewrite the sum as

$$
\sum_{r_{x} \in \mathbb{F}_{p}^{\times}} \omega^{-a}\left(r_{x}\right) \zeta_{\pi}^{\omega\left(r_{x}\right)}=\sum_{r_{x} \in \mathbb{F}_{p}^{\times}} \omega^{-a}\left(r_{x}\right) \zeta_{\pi}^{r_{x}},
$$

since $\omega\left(r_{x}\right) \equiv r_{x}(\bmod \mathfrak{p})$. The Gross-Koblitz formula becomes

$$
-\sum_{x \in \mathbb{F}_{p}} \omega^{-a}(x) \zeta_{p}^{x}=\pi^{a} \Gamma_{p}\left(\frac{a}{p-1}\right)
$$

Remark 4. The Gross-Koblitz formula for $f=1$, $p$ odd implies Stickelberger's Theorem (Theorem 2.6). To see this, we need to show $\Gamma_{p}\left(\frac{a}{p-1}\right) \equiv \frac{1}{a !}(\bmod p)$ for $0 \leq a<p-1$. From the relations $\frac{1}{1-p}=1+p+p^{2}+\ldots$ and $-a=(p-a)(1-p)+p(p-a-1)$, we 
see that $\frac{a}{p-1}=\frac{-a}{1-p}=p-a+(p-a-1)\left(p+p^{2}+p^{3}+\ldots\right)$. We have

$$
\begin{aligned}
\Gamma_{p}\left(\frac{a}{p-1}\right) & =\Gamma_{p}\left(\frac{-a}{1-p}\right) \equiv \Gamma_{p}(p-a) \quad(\bmod p) \\
& =(-1)^{p-a} \prod_{\substack{j<p-a \\
p \nmid j}} j=(-1)^{p-a}(p-a-1) !
\end{aligned}
$$

where the above follows from Lemma 3.4 by choosing $\left\{k_{i}\right\}$ to be the sequence of partial sums of the $p$-adic expansion of $\frac{a}{p-1}$. From $(p-1) ! \equiv-1(\bmod p)$,

$$
(p-a-1) ! \equiv \frac{-1}{(p-1)(p-2) \cdots(p-a)} \quad(\bmod p) \equiv \frac{(-1)^{1-a}}{a !} \quad(\bmod p) .
$$

Finally, $\Gamma_{p}\left(\frac{a}{p-1}\right) \equiv(-1)^{p-a} \cdot \frac{(-1)^{1-a}}{a !}(\bmod p)=\frac{1}{a !}(\bmod p)$.

\subsection{Estimates of Solutions of Norm Equations}

Recall that the ring of integers for $\mathbb{Q}(\sqrt{-d})$ is $\mathbb{Z}\left[\frac{1+\sqrt{-d}}{2}\right]$ if $d \equiv 3(\bmod 4)$ and $\mathbb{Z}[\sqrt{-d}]$ if $d \equiv 1,2(\bmod 4)$. We have

$$
\left(x+\frac{1+\sqrt{-d}}{2} y\right)\left(x+\frac{1-\sqrt{-d}}{2} y\right)=\frac{(2 x+y)^{2}}{4}+\frac{d y^{2}}{4}
$$

and

$$
(x+\sqrt{-d} y)(x-\sqrt{-d} y)=x^{2}+d y^{2} .
$$


In both cases, we want the right side of the equation to be $p^{h}$, with $p$ splitting in $\mathbb{Q}(\sqrt{-d})$ and $y \not \equiv 0(\bmod p)$. We estimate the values of $x$ and $y$ as follows.

Proposition 3.6. Let $p$ be a prime (including $p=2$ ) that splits in $\mathbb{Q}(\sqrt{-d})$. Let $x, 0 \neq y \in$ $\mathbb{Z}$ be such that

$$
\begin{aligned}
(2 x+y)^{2}+d y^{2} & =4 p^{h}, \quad \text { if } d \equiv 3 \quad(\bmod 4) \\
x^{2}+d y^{2} & =p^{h}, \quad \text { if } d \equiv 1,2 \quad(\bmod 4) .
\end{aligned}
$$

Let $b=2 x+y$ or $b=x$, respectively. Then, except the cases when $d=3, p=7$ or 13 ; $d=11, p=5$,

$$
|b| \leq \frac{p^{h}}{2}
$$

Proof. Let $a=p^{h}$. We first consider the case $d \equiv 1,2(\bmod 4)$. Suppose equation (3.2) holds. Then $b^{2}=a-d y^{2} \leq a-d$, so inequality (3.3) holds if $a-d \leq \frac{a^{2}}{4} \Leftrightarrow a^{2}-4 a+4 d \geq$ 0 . This is true since $a^{2}-4 a+4 d \geq a^{2}-4 a+4=(a-2)^{2} \geq 0$.

We now consider the case $d \equiv 3(\bmod 4)$. If equation $(3.1)$ holds, then $b^{2}=4 a-d y^{2} \leq$ $4 a-d$, so inequality (3.3) holds if $4 a-d \leq \frac{a^{2}}{4} \Leftrightarrow a^{2}-16 a+4 d \geq 0 \Leftrightarrow(a-8)^{2}+4 d \geq$ $64 \Leftrightarrow 64-4 d \leq 0$ or $d<16$ and $a \geq 8+\sqrt{64-4 d}$ or $a \leq 8-\sqrt{64-4 d}$. So it remains only to examine the cases when $d=3,7,11,15$ and $8-\sqrt{64-4 d}<a<8+\sqrt{64-4 d}$.

If $d=3$ then $h=1$, so the possible $p$ 's are such that $2 \leq p<15$. For $d=7, h=1$ and the possible $p$ 's are $2<p<14$; for $d=11, h=1$ so $3<p<12$; for $d=15, h=2$ so 
$6<p^{2}<10 \Leftrightarrow p=3$, but since 3 divides the discriminant, 3 ramifies in $\mathbb{Q}(\sqrt{-15})$. Let us consider each case.

If $d=3, p$ may be 7 or 13 because $p$ must be congruent to $1(\bmod 3)$. For $p=7$, the solution is $b=5, y=1$. For $p=13$, the solution is $b=7, y=1$. Therefore, inequality (3.3) fails.

If $d=7$, the condition $\left(\frac{-7}{p}\right)=1$ leaves only $p=11$. The only solutions of $b^{2}+7 y^{2}=$ 44 are $( \pm 4, \pm 2)$, so inequality (3.3) holds.

If $d=11$, the condition $\left(\frac{-11}{p}\right)=1$ leaves only $p=5$. The solutions of $b^{2}+11 y^{2}=20$ are $( \pm 3, \pm 1)$, so inequality (3.3) fails.

Suppose that $x$ and $y$ is a solution of equation (3.1) or (3.2). Suppose $d \equiv 3(\bmod 4)$. Defining $\alpha$ to be $2 x+y\left(\bmod p^{h}\right)$ and letting $\langle\alpha\rangle$ be defined by Definition 2.7 but with $n$ replaced by $p^{h}$, if $p \neq 7,13$ for $d=3$ and $p \neq 5$ for $d=11$, we get

$$
y= \pm \sqrt{\frac{4 p^{h}-\langle\alpha\rangle^{2}}{d}}
$$

and

$$
x=\frac{\langle\alpha\rangle-y}{2}
$$

When $d \equiv 1,2(\bmod 4)$, define $\alpha \equiv x\left(\bmod p^{h}\right)$ so that Proposition 3.6 implies that

$$
x=\langle\alpha\rangle,
$$


and

$$
y= \pm \sqrt{\frac{p^{h}-\langle\alpha\rangle^{2}}{d}}
$$

Remark 5. If $\left(x_{0}, y_{0}\right)$ is a solution to the norm equation (3.1) or (3.2), then $\left(-x_{0},-y_{0}\right)$ is also a solution to the norm equation. Thus, we may replace $\langle-\alpha\rangle$ with $\langle\alpha\rangle$ in our computations and the resulting $x$ and $y$ that we find are still solutions.

Proposition 3.7. Let $k=k(h)=\left\{\begin{array}{cc}\frac{h}{2}+1 & \text { if } h \text { is even } \\ \frac{h}{2}+\frac{1}{2} & \text { if } h \text { is odd }\end{array}\right.$. Under the conditions of Proposition 3.6, for $d \neq 1,2$ we have that

$$
|b| \leq \frac{p^{c}}{2}
$$

where for $d \equiv 1,2(\bmod 4), c=k ;$ for $d \equiv 3(\bmod 4), c=k$ if $h \leq 2$, also $c=k$ if $d$ is not a prime, $p \geq 5$ or $p \leq 3$ and $d \geq p^{h}\left(\frac{16-p^{2}}{4}\right)$, also $c=k$ if $d$ is a prime, $p \geq 17$ or $p \leq 13$ and $d \geq p^{h}\left(\frac{16-p}{4}\right)$. Otherwise, for $d \equiv 3(\bmod 4), c=k+1$, except the case when $d$ is prime, $h \geq 5, p=2, d<2^{h+1}$ when $c=k+2$.

Proof. If $h \leq 2, k=h$, so the estimate with $c=k$ is the same as in Proposition 3.6.

Note that $2 k=h+2$ if $h$ is even and $2 k=h+1$ if $h$ is odd.

It is known ([1], Chapter 3, Section 8, Theorem 8) that $h$ is even if and only if the number of distinct prime divisors of the discriminant $D$ is bigger than 1 . So if $d \equiv 1,2$ 
$(\bmod 4), d \neq 1,2$, then $h$ is even, so

$$
\frac{p^{2 k}}{4}=\frac{p^{h} p^{2}}{4} \geq p^{h} \geq|b|^{2}
$$

and the estimate with $c=k$ follows.

Now let $d \equiv 3(\bmod 4)$. Recall that $4 p^{h}-d \geq|b|^{2}$ in this case. If $d$ is not a prime, then $h$ is even, so

$$
\frac{p^{2 k}}{4}=\frac{p^{h} p^{2}}{4} \geq 4 p^{h}-d
$$

if $p \geq 5$ or $p \leq 3$ and $d \geq p^{h}\left(\frac{16-p^{2}}{4}\right)$ and the estimate with $c=k$ follows. Also,

$$
\frac{p^{2(k+1)}}{4}=\frac{p^{h} p^{4}}{4} \geq 4 p^{h}
$$

and the estimate with $c=k+1$ follows for remaining cases with $d \equiv 3(\bmod 4), d$ not a prime.

If $d$ is a prime, then $h$ is odd, so

$$
\frac{p^{2 k}}{4}=\frac{p^{h} p}{4} \geq 4 p^{h}-d
$$

if $p \geq 17$ or $2 \leq p \leq 13$ and $d>p^{h}\left(\frac{16-p}{4}\right)$ and the estimate with $c=k$ follows. Also,

$$
\frac{p^{2(k+1)}}{4}=\frac{p^{h} p^{3}}{4} \geq 4 p^{h}-d
$$

if $p \geq 3$ or $p=2$ and $d \geq 2^{h+1}$, so the estimate with $c=k+1$ holds. 
If $p=2, d$ is a prime, $d<2^{h+1}$, then the estimate with $c=k+2$ holds because

$$
\frac{2^{2(k+2)}}{4}=\frac{2^{h} 2^{5}}{4}>4\left(2^{h}\right)
$$

Note also that if $h=3$, then $k+1=h$, so the estimate with $c=k+1$ follows from Proposition 3.6.

\subsection{The Class Number Formulas}

The following statements will be used throughout the paper.

Since $d$ is not necessarily prime, we need to modify the definition of the Legendre symbol $\left(\frac{a}{d}\right)$.

Definition 3.8. Let $d=p_{1}^{\alpha_{1}} p_{2}^{\alpha_{2}} \ldots p_{n}^{\alpha_{n}}$ be an positive odd integer and let a be any integer. We define the Jacobi symbol to be

$$
\left(\frac{a}{d}\right)=\left(\frac{a}{p_{1}}\right)^{\alpha_{1}}\left(\frac{a}{p_{2}}\right)^{\alpha_{2}} \cdots\left(\frac{a}{p_{n}}\right)^{\alpha_{n}}
$$

where $\left(\frac{a}{p_{i}}\right)$ is the Legendre symbol.

The Jacobi symbol has properties similar to that of the Legendre symbol. Like the Legendre symbol, if $\left(\frac{a}{d}\right)=-1$, then $a$ is a quadratic non residue modulo $d$. However, no conclusion can be made if $\left(\frac{a}{d}\right)=1$. 
It can be shown as a consequence of a lemma of Gauss that

$$
\left(\frac{2}{d}\right)=\left\{\begin{array}{rll}
1 & \text { if } d \equiv 1,7 \quad(\bmod 8) \\
-1 & \text { if } d \equiv 3,5 \quad(\bmod 8)
\end{array} .\right.
$$

(See page 53 of [5], for example.)

From the Kronecker-Weber theorem, we have

Proposition 3.9. Let $d$ be a square-free positive integer. If $d \equiv 3(\bmod 4)$, set $m=d$. Otherwise, set $m=4 d$. Then $\mathbb{Q}(\sqrt{-d}) \subseteq \mathbb{Q}\left(\zeta_{m^{\prime}}\right)$ if and only if $m^{\prime}$ is a multiple of $m$. Thus, $m$ is the smallest positive integer $m^{\prime}$ for which the inclusion holds.

The proof of this statement can be found in [6].

Proposition 3.10. For an imaginary quadratic field with discriminant $D<-4$, we have the formula

$$
\begin{gathered}
h=-\frac{1}{|D|} \sum_{\substack{(a, D)=1 \\
0<a<|D|}} \chi_{d}(a) a, \\
0<
\end{gathered}
$$

where $\chi_{d}$ is the character of $\mathbb{Q}(\sqrt{-d})$ defined by

$$
\chi_{d}(a)= \begin{cases}\left(\frac{a}{d}\right) & \text { if } d \equiv 3 \quad(\bmod 4) \\ (-1)^{\frac{a-1}{2}}\left(\frac{a}{d}\right) & \text { if } d \equiv 1 \quad(\bmod 4) \\ (-1)^{\frac{a^{2}-1}{8}+\frac{a-1}{2} \cdot \frac{-d^{\prime}-1}{2}}\left(\frac{a}{d^{\prime}}\right) & \text { if } d=2 d^{\prime}\end{cases}
$$


for $(a, D)=1$ and $\chi_{d}(a)=0$ for $(a, D) \neq 1$. Note that

$$
D= \begin{cases}-d & \text { if } d \equiv 3 \quad(\bmod 4) \\ -4 d & \text { if } d \equiv 1,2 \quad(\bmod 4)\end{cases}
$$

(see [1], page 344).

The following from page 346 of [1] will be used:

Theorem 3.11. For an imaginary quadratic field with discriminant $D<-4$ and character $\chi_{d}$ we have

$$
h=\frac{1}{2-\chi_{d}(2)} \sum_{\substack{0<a<\frac{|D|}{2} \\(a, D)=1}} \chi_{d}(a) .
$$

Note that when $D$ is even, $\chi_{d}(2)=0$.

In particular, when the field is $\mathbb{Q}(\sqrt{-\ell})$, where $\ell$ is prime, we have

Theorem 3.12. Let $\ell$ be a prime number, $\ell \equiv 3(\bmod 4), \ell \neq 3$. Let $V$ and $N$ denote the number of quadratic residues and nonresidues in the interval $\left(0, \frac{\ell}{2}\right)$. The number of divisor classes of the field $\mathbb{Q}(\sqrt{-\ell})$ is odd and is given by

$$
\begin{array}{lll}
h=V-N & \text { for } \ell \equiv 7 & (\bmod 8) \\
h=\frac{1}{3}(V-N) & \text { for } \ell \equiv 3 & (\bmod 8)
\end{array}
$$

\subsection{Representing an Element in $\mathbb{Q}(\sqrt{-d})$ with Norm $p^{h}$ as a Product of Gauss Sums}

We now give the factorization of the Gauss and Jacobi sums. Let $L=\mathbb{Q}\left(\zeta_{m}\right)$ and $m$ be as in section 2.1. Let $\wp_{1}$ be a prime of $\mathbb{Q}\left(\zeta_{m}\right)$ lying above $p$. Let $\omega$ be the Teichmüller 
character from $\mathbb{F}_{q}^{\times}$to $\mu_{q-1}$ corresponding to a prime of $\mathbb{Q}\left(\zeta_{q-1}\right)$ lying above $\wp_{1}$, and let $\chi=\omega^{c}$, where $c=\frac{q-1}{m}$. We have

Proposition 3.13 (Stickelberger's Relations). Let $\wp_{1}$ be a prime of $\mathbb{Q}\left(\zeta_{m}\right)$ lying above p.

Then

1. $\left(\tau_{p}\left(\chi^{-1}\right)^{m}\right)=\wp_{1}^{m \theta}=\wp_{1}^{1 \leq a<m,(a, m)=1}{ }^{a \sigma_{a}^{-1}}=\prod_{1 \leq a<m,(a, m)=1}\left(\wp_{1}^{\sigma_{a}^{-1}}\right)^{a}$,

as ideals in $\mathbb{Q}\left(\zeta_{m}\right)$.

2. Let $j, k$ be integers such that $j, k, j+k \not \equiv 0(\bmod m)$ and define

$$
\theta_{j, k}=\sum_{\substack{1 \leq a<m \\(a, m)=1}}\left(\left\lfloor\frac{(j+k) a}{m}\right\rfloor-\left\lfloor\frac{j a}{m}\right\rfloor-\left\lfloor\frac{k a}{m}\right\rfloor\right) \sigma_{a}^{-1} .
$$

Then $\left(J\left(\chi^{-j}, \chi^{-k}\right)\right)=\wp_{1}^{\theta_{j, k}}$ as ideals in $\mathbb{Q}\left(\zeta_{m}\right)$.

Proof. See [7].

Let $K=\mathbb{Q}(\sqrt{-d})$, where $d>0$ is square free. Suppose $\left(\frac{D}{p}\right)=1$, where $D$ is the discriminant of the field. Then $p \mathscr{O}_{K}=\delta_{1} \delta_{2}$. By Proposition 3.9, we see that $K \subset L$, where $L=\mathbb{Q}\left(\zeta_{|D|}\right)$

Let $f$ be the order of $p$ in $(\mathbb{Z} /|D| \mathbb{Z})^{\times}$, so $p^{f} \equiv 1(\bmod |D|)$. In this case, we have $p \mathscr{O}_{L}=\wp_{1} \wp_{2} \cdots \wp_{g}$, where $g=\frac{\varphi(D)}{f}$ (see [14], Theorem 2.13, page 14). Let $M$ be the maximal subfield of $L$ containing $\mathbb{Q}$ in which $p$ splits completely. $M$ is the fixed field of the decomposition group of $\wp_{i}$ (see [10], page 17), which is the subgroup of $\mathrm{Gal}(L / \mathbb{Q})$ 
generated by $\sigma_{p}$. Now, $[L: M]=f$ and $[M: \mathbb{Q}]=\frac{\varphi(|D|)}{f}$. Thus, each prime lying above $p$ in $M$ has one prime lying above it in $L$ and the residue degree is $f$. Let $p \mathscr{O}_{M}=\mathcal{D}_{1} \mathcal{D}_{2} \cdots \mathcal{D}_{g}$ with each $\wp_{i}$ lying above $\mathcal{D}_{i}$. Then $N_{L / M}\left(\wp_{i}\right)=\mathcal{D}_{i}^{f}$.

Let $G_{n}=\operatorname{Gal}\left(\mathbb{Q}\left(\zeta_{n}\right) / \mathbb{Q}\right)$. The map $b \mapsto \sigma_{b}$, where $\sigma_{b}\left(\zeta_{n}\right)=\zeta_{n}^{b}$ gives a canonical isomorphism of $(\mathbb{Z} / n \mathbb{Z})^{\times}$and $G_{n}$. Let $(t, n)=1$. Then $G_{n t}=G_{n} \times G_{t}$ with $\mathbb{Q}\left(\zeta_{t}\right), \mathbb{Q}\left(\zeta_{n}\right)$ being fixed fields for $G_{n}, G_{t}$, respectively. It follows from Galois theory that if $H \subset G_{n}$ and $F=\mathbb{Q}\left(\zeta_{n}\right)^{H}$, then $\mathbb{Q}\left(\zeta_{n t}\right)^{H}=F\left(\zeta_{t}\right)$.

$$
\begin{aligned}
& \text { Let } m=|D|, q=p^{f}, \chi: \mathbb{F}_{q} \rightarrow \mu_{m} \text { be a character. Then } \\
& \sigma_{p}\left(-\tau_{p}(\chi)\right)=\sigma_{p}\left(\sum \chi(a) \zeta_{p}^{\operatorname{Tr}(a)}\right)=\sum \chi\left(a^{p}\right) \zeta_{p}^{\operatorname{Tr}(a)}=\sum \chi\left(a^{p}\right) \zeta_{p}^{\operatorname{Tr}\left(a^{p}\right)}=-\tau_{p}(\chi) .
\end{aligned}
$$

Since the fixed field for $\left\langle\sigma_{p}\right\rangle$ in $L$ is $M$, this implies that $\tau_{p}(\chi) \in M\left(\zeta_{p}\right)$.

Let $\chi=\omega^{\frac{q-1}{m}}$, where $\omega$ is the Teichmüller character associated to a prime of $\mathbb{Q}\left(\zeta_{q-1}\right)$ above $\wp_{1}$. Define $W=N_{M\left(\zeta_{p}\right) / K\left(\zeta_{p}\right)}\left(\tau_{p}(\chi)\right) \in K\left(\zeta_{p}\right)$. We will prove that $W \in \mathscr{O}_{K}$ and for some $\eta \in \mathbb{N}$, we have $Z=W / p^{\eta} \in \mathscr{O}_{K}$ and $N_{K / \mathbb{Q}}(Z)=p^{h}$. Therefore, $Z$ will serve for our purpose of finding solutions of the norm equations in the general case $(d \neq 1,2,3)$.

Let $H \subset G_{m}$ denote Gal $(L / K)$. Let $R$ be a set of representatives of $H /\langle p\rangle$ in the set of integers in the interval $(0, m)$. Then $W=\prod_{b \in R} \tau_{p}\left(\chi^{b}\right)$. Let $\psi=\left.\left(\prod_{b \in R} \chi^{b}\right)\right|_{\mathbb{F}_{p}}$. We note that for $c \in(\mathbb{Z} / p \mathbb{Z})^{\times}$, the action of $g_{c} \in \operatorname{Gal}\left(L\left(\zeta_{p}\right) / L\right)$ on $W$ is given by

$$
g_{c}(W)=\psi^{-1}(c) W
$$


In fact, we have

$$
g_{c}\left(\tau_{p}\left(\chi^{b}\right)\right)=-\sum \chi^{b}(a) \zeta_{p}^{c \operatorname{Tr}(a)}=-\sum \chi^{b}\left(a c c^{-1}\right) \zeta_{p}^{\operatorname{Tr}(c a)}=\chi^{-b}(c) \tau_{p}\left(\chi^{b}\right)
$$

Thus, to show that $W \in K$, we need to show that $\psi=\psi_{0}$.

Let $[a]_{m}$ be the integer representing $a$ in the interval $[0, m)$. Let $i=\sum_{a \in H \subset(\mathbb{Z} / m \mathbb{Z})^{\times}}[a]_{m}$.

We will show that $\psi=\psi_{0}$ if $i \equiv 0(\bmod m)$. In fact, $\psi=\psi_{0}$ if and only if $\frac{q-1}{m}\left(\sum_{b \in R} b\right) \equiv 0(\bmod p-1)$ because $\left.\omega\right|_{\mathbb{F}_{p}}$ has strict order $p-1$. But

$$
\frac{q-1}{m}\left(\sum_{b \in R} b\right)=(p-1) \frac{\left(1+p+\cdots+p^{f-1}\right)\left(\sum_{b \in R} b\right)}{m} \equiv 0 \quad(\bmod p-1)
$$

because

$$
\left(1+p+\cdots+p^{f-1}\right)\left(\sum_{b \in R} b\right) \equiv i \quad(\bmod m) \equiv 0 \quad(\bmod m)
$$

Proposition 3.14. If $d \neq 1,2,3($ respectively, $m \neq 4,8,3)$, then $i \equiv 0(\bmod m)$.

Proof. We note that $m$ is either a power of a prime, or a product of $m_{1} m_{2}$, where $\left(m_{1}, m_{2}\right)=$ 1 and $m_{i}>2$ for $i=1,2$. In the first case, $m=p \equiv 3(\bmod 4)$ and the prime $p$ is greater than 3. Note that $4(\bmod m) \in H$. Hence, $H=4 H$ and

$$
i=\sum_{h \in H}[4 h]_{m} \equiv 4 \sum_{h \in H}[h]_{m} \equiv 4 i \quad(\bmod p)
$$

So $3 i \equiv 0(\bmod p)$ implies $i \equiv 0(\bmod p)$. 
Now we consider the case $m=m_{1} m_{2}$. Let $n$ be one of $m_{1}, m_{2}$. Let $\gamma: G_{m} \rightarrow G_{n}$ be the natural surjection. Note that $K \not \subset \mathbb{Q}\left(\zeta_{n}\right)$ by Proposition 3.9, so ker $\gamma \not \subset H$ by Galois theory. So there exists $c \in \operatorname{ker} \gamma, c \notin H$. Then $G_{m}=c H \cup H$ because $H$ has index 2 in $G_{m}$.

We conclude that $G_{n}=\gamma\left(G_{m}\right)=\gamma(c H) \cup \gamma(H)=(\gamma(c) \gamma(H)) \cup \gamma(H)=\gamma(H)$ since $\gamma(c)=1$.

Note that $\sum_{b \in G_{n}}[b]_{n}=\frac{1}{2}\left(\sum_{b \in G_{n}}[b]_{n}+\sum_{b \in G_{n}}\left(n-[b]_{n}\right)\right)=\frac{1}{2} n \varphi(n)$, where $\varphi(n)=\left|G_{n}\right|$. So $\sum_{b \in G_{n}}[b]_{n} \equiv 0(\bmod n)$ if $n>2$.

We have $i(\bmod n)=\sum_{h \in H} \gamma(h)=\left[H / G_{n}\right]\left(\sum_{b \in G_{n}} b\right) \equiv 0(\bmod n)$. So $i \equiv 0\left(\bmod m_{1}\right)$, $i \equiv 0\left(\bmod m_{2}\right)$ and $i \equiv 0\left(\bmod m_{1} m_{2}\right)$ because $\left(m_{1}, m_{2}\right)=1$.

Since $i \equiv 0(\bmod m)$, then $\psi=\psi_{0}$.

Now let $\eta=\frac{i}{m} \in \mathbb{N}$. We wish to determine the factorization of $W \in K$ into prime divisors of $K$.

Note that $\tau_{p}\left(\chi^{-1}\right)^{m} \in L$ and by Proposition 3.13,

$$
\left(\tau_{p}\left(\chi^{-1}\right)^{m}\right)=\wp_{1}^{\theta}, \quad \text { where } \theta=\sum_{\substack{1 \leq a<m \\(a, m)=1}} a \sigma_{a}^{-1}
$$

Note that $\overline{\tau_{p}(\chi)}=\chi(-1) \tau_{p}\left(\chi^{-1}\right)$, so

$$
\bar{W}=\left(\tau_{p}\left(\chi^{-1}\right) \chi(-1)\right)^{\sum_{b \in R} \sigma_{b}}=\tau_{p}\left(\chi^{-1}\right)^{\sum \sigma_{b}} \psi(-1)=\tau_{p}\left(\chi^{-1}\right)^{\sum \sigma_{b \in R}}
$$


because $\psi=\psi_{0}$. Therefore,

$$
\left(\bar{W}^{m}\right)=\wp_{1}{ }^{\theta}\left(\sum_{b \in R} \sigma_{b}\right)=\left(\wp_{1} \sigma_{1} \sigma_{b}\right)^{\theta} .
$$

However, $\wp_{1} \sigma_{1} \sigma_{b}$ is the image of a prime divisor $\delta_{1}$ of $K$ above $p$ into the group of divisors of $L$. Since the action of $\sigma_{a}$ on the image of $\delta_{1}$ depends only on $\left.\sigma_{a}\right|_{K}$,

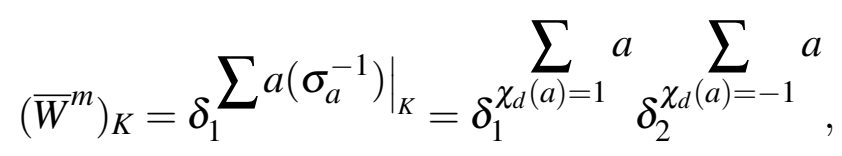

where $\delta_{2}=\overline{\delta_{1}}$ is the conjugate of $\delta_{1}$, so $\delta_{1} \delta_{2}=(p)$. Therefore,

$$
(\bar{W})_{K}^{m}=\delta_{2}^{-\sum \chi_{d}(a) a_{\left(\delta_{2} \delta_{1}\right) \chi_{d}(a)=1} a}=\delta_{2}^{-\sum \chi_{d}(a) a}(p)^{m \eta} .
$$

Taking into account that by Proposition 3.10, $h=-\frac{1}{m} \sum \chi_{d}(a) a$, we get that

$$
(\bar{W})_{K}=\delta_{2}^{h}(p)^{\eta}
$$

Finally, $Z=W / p^{\eta} \in \mathscr{O}_{K}$ and $(Z)=\delta_{1}^{h}$. See section 3.6 for a representation of $W$ as a sum of the $m$-th roots of unity. Also, note that the equality $|Z|^{2}=p^{h}$ implies that $\eta=\frac{\varphi(m) / 2-h}{2}$. 


\subsection{Solutions of Norm Equations in the case $\mathbb{Q}(\sqrt{-d}), d>$ $0, d$ square-free, $d \neq 1,2,3,\left(\frac{D}{p}\right)=1$}

Let $x+w y=Z=\frac{\prod_{b \in R} \tau_{p}\left(\omega^{\frac{q-1}{m} b}\right)}{p^{\eta}}$, where

$$
w=\left\{\begin{array}{ll}
\sqrt{-d} & \text { if } d \equiv 1,2 \quad(\bmod 4) \\
\frac{1+\sqrt{-d}}{2} & \text { if } d \equiv 3 \quad(\bmod 4)
\end{array} .\right.
$$

We showed that $N_{K / \mathbb{Q}}(Z)=p^{h}$ and $(Z)=\delta_{1}^{h}$.

Let $[e]_{n}$ denote, as usual, the integer in $[0, n)$ representing $e(\bmod n)$. We want to express $\bar{Z}$ as a product of values of $p$-adic Gamma function using the Gross-Koblitz formula. We have

$$
\bar{Z}=\frac{\prod_{b \in R} \tau_{p}\left(\omega^{-\frac{q-1}{m} b}\right)}{p^{\eta}}=\frac{\pi^{v}}{p^{\eta}} \prod_{b \in R} \prod_{k=0}^{f-1} \Gamma_{p}\left(\frac{\left[\frac{q-1}{m} b\right]_{q-1}^{(k)}}{q-1}\right),
$$

where $a^{(k)}$ in the Gross-Koblitz formula equals $\left[a p^{k}\right]_{q-1}$, so $\left[\frac{q-1}{m} b\right]_{q-1}^{(k)}=\frac{q-1}{m}\left[b p^{k}\right]_{m}$.

We note that $\frac{\pi^{v}}{p^{\eta}}=(-1)^{\eta}$ because $\bar{Z}$ and the product of values of $\Gamma_{p}$ are $\delta_{1}$-units, so $v=(p-1) \eta$ and $\frac{\pi^{v}}{p^{\eta}}=\left(\frac{\pi^{p-1}}{p}\right)^{\eta}=(-1)^{\eta}$.

Because the set $\left\{b p^{k}(\bmod m): b \in R, k=0,1, \ldots, f-1\right\}=$

$$
\begin{aligned}
&\left\{a \in(\mathbb{Z} / m \mathbb{Z})^{\times}: \chi_{d}(a)=1\right\} \text {, we have } \\
& \bar{Z}=(-1)^{\eta} \prod_{\substack{1 \leq a<m \\
\chi_{d}(a)=1}} \Gamma_{p}\left(\frac{a}{m}\right)=\beta .
\end{aligned}
$$


We also have

$$
\begin{aligned}
& x+w y \equiv 0 \quad\left(\bmod \delta_{1}^{h}\right), \\
& \overline{x+w y}=\beta .
\end{aligned}
$$

Therefore,

$$
\begin{aligned}
x & \equiv \frac{1}{2} \beta \quad\left(\bmod p^{h}\right) \quad \text { when } d \equiv 1,2 \quad(\bmod 4), \\
2 x+y & \equiv \beta \quad\left(\bmod p^{h}\right) \quad \text { when } d \equiv 3 \quad(\bmod 4) .
\end{aligned}
$$

Let $\langle e\rangle_{p^{n}}$ denote the integer in the interval $\left(-\frac{p^{n}}{2}, \frac{p^{n}}{2}\right]$ representing $e\left(\bmod p^{n}\right)$. Let $c \leq n \leq h$, where $c$ is defined in Proposition 3.7 in section 3.2. Let $[e]=[e]_{p^{n}},\langle e\rangle=\langle e\rangle_{p^{n}}$. Let $T=\left[\frac{1}{m}\right]$ and $\alpha=(-1)^{\eta} \prod_{\substack{1 \leq a<m \\ \chi_{d}(a)=1}}(-1)^{[a T]} \prod_{\substack{1 \leq j<[a T] \\(j, p)=1}} j$. We have

Theorem 3.15. Let $d \neq 1,2,3$ and suppose $p$ splits in $K=\mathbb{Q}(\sqrt{-d})$. Assume $p \neq 2$ if $d=7$ and $p \neq 5$ if $d=11$. If $d \equiv 1,2(\bmod 4)$, then $x=\left\langle\frac{1}{2} \beta\right\rangle=\left\langle\frac{1}{2} \alpha\right\rangle, y= \pm \sqrt{\frac{p^{h}-x^{2}}{d}}$ are solutions in $\mathbb{Z}, p \nmid y$, of $x^{2}+d y^{2}=p^{h}$. If $d \equiv 3(\bmod 4)$, then $2 x+y=\langle\beta\rangle=\langle\alpha\rangle$ (if $p=2,\langle\beta\rangle=\langle\alpha\rangle$ if $n \neq 2), y= \pm \sqrt{\frac{4 p^{h}-(2 x+y)^{2}}{d}}$ are solutions in $\mathbb{Z}$, with $x, y$ not both divisible by $p$, of $(2 x+y)^{2}+d y^{2}=4 p^{h}$.

Proof. We know that $p$ does not divide both $x$ and $y$ because otherwise $p$ divides $Z$, but $(Z)=\delta_{1}^{h}$

Let $d \equiv 1,2(\bmod 4)$. Then $p \neq 2($ since 2 ramifies in $K)$, so $|x| \neq \frac{p^{n}}{2}$ because $x \in \mathbb{Z}$. 
Also, $|x| \leq \frac{p^{n}}{2}$ according to Proposition 3.7. Then $\left|x-\left\langle\frac{1}{2} \beta\right\rangle\right|<\frac{p^{n}}{2}+\frac{p^{n}}{2}$ and we have shown (see above) that $p^{n} \mid\left(x-\left\langle\frac{1}{2}\right\rangle \beta\right)$, so $x=\left\langle\frac{1}{2} \beta\right\rangle$.

The proof is the same for the case $d \equiv 3(\bmod 4)$ if we show that $|b| \neq \frac{p^{n}}{2}$, where $b=2 x+y=\langle\beta\rangle .|b| \neq \frac{p^{n}}{2}$ is true if $p \neq 2$. If $p=2$, then $d \equiv 7(\bmod 8)$ because 2 splits in $K$ and we have the norm equality $b^{2}+d y^{2}=4\left(2^{h}\right)$. In particular, $1+d \leq 4\left(2^{h}\right)$. If $h \geq 4$, then $c \geq k \geq 3$ (see the proof of Proposition 3.7), so $n \geq c \geq 3$. If $|b|=\frac{2^{n}}{2}=2^{n-1}$, then $4|b \Rightarrow 4| y \Rightarrow 2 \mid x$, a contradiction. If $h=1$, then $d=7$, but this case is excluded. If $h=2$, then $d=15, b= \pm 1$. If $h=3$, then $d=23$ or 31 and $b= \pm 3$ or $b= \pm 1$, respectively. Thus, if $h=2$ or 3 , then $n \geq c \geq k \geq 2$, so $|b| \neq \frac{2^{n}}{2}$.

Now, $\Gamma\left(\frac{a}{m}\right) \equiv \Gamma\left(\left[\frac{a}{m}\right]\right)=\Gamma([a T])\left(\bmod p^{n}\right)$ and $\Gamma(k)=(-1)^{k} \prod_{\substack{1 \leq j<k \\(j, p)=1}} j$, according to the properties of the $p$-adic Gamma functions in section 3.1.

We note that one can remove $(-1)^{*}$ from the expressions for $\beta$ and $\alpha$, then we get

$$
( \pm x, y),( \pm(2 x+y), y)
$$

instead of

$$
(x, y),(2 x+y, y)
$$

(because $\langle-e\rangle=-\langle e\rangle$ ), which are also solutions of the norm equations. 


\subsection{Solutions of Norm Equations as Normalized Trace of a Ratio of Products of Gauss Sums}

In this section, we describe how to compute $x$ when $d \equiv 1,2(\bmod 4)$ and $x+\frac{y}{2}$ when $d \equiv 3(\bmod 4)$ by applying the normalized trace to $Z$ or its image in $\mathbb{F}_{q}$.

Let $G$ be an abelian group with elements of finite order. Define a function $t: G \rightarrow \mathbb{Q}$ as follows: let $g \in G$ and $n(g)$ be the strict order of $g$. Then

$$
t(g)=\mu(n(g)) \prod_{\ell \mid n(g)} \frac{1}{\ell-1}
$$

where $\ell$ runs through all primes dividing $n(g)$ and $\mu(n)$ is the Möbius function defined by

$$
\mu(n)= \begin{cases}0 & \text { if } n \text { is not square-free } \\ \prod_{\ell \mid n}(-1) & \text { otherwise }\end{cases}
$$

In particular, we have defined $t$ on the group of roots of unity in $\overline{\mathbb{Q}}$.

Let $a$ be an algebraic number. Define the normalized trace by $\operatorname{Tr}_{0}(a)=\frac{\operatorname{Tr}_{F / \mathbb{Q}}(a)}{\operatorname{deg}(F / \mathbb{Q})}$, where $F$ is any finite extension of $\mathbb{Q}$ containing $a$. Note that $\operatorname{Tr}_{0}(a)$ does not depend on the choice of $F$.

If $\zeta$ is a root of unity, then $\operatorname{Tr}_{0}(\zeta)=t(\zeta)$. To see this, let $n=\operatorname{order}(\zeta)$ and let $n=\prod_{i} q_{i}$ be the decomposition of $n$ into product of powers of distinct primes. The group $\operatorname{Gal}(\mathbb{Q}(\zeta) / \mathbb{Q})=\prod_{i} \operatorname{Gal}\left(\mathbb{Q}\left(\zeta_{q_{i}}\right) / \mathbb{Q}\right)$ and $\operatorname{Tr}_{0}(\zeta)=\prod_{i} \operatorname{Tr}_{0}\left(\zeta_{q_{i}}\right)$. However, 
$\operatorname{Tr}_{\mathbb{Q}\left(\zeta_{q_{i}}\right) / \mathbb{Q}}\left(\zeta_{q_{i}}\right)=0$ if $q_{i}=\ell^{k}, k \geq 2$ because

$$
\operatorname{Tr}_{\mathbb{Q}\left(\zeta_{\ell^{k}}\right) / \mathbb{Q}\left(\zeta_{\ell^{k-1}}\right)}\left(\zeta_{\ell^{k}}\right)=\sum_{j=0}^{\ell-1} \zeta_{\ell^{k}}^{1+\ell^{k-1} j}=\zeta_{\ell^{k}}\left(\sum_{j=0}^{\ell-1} \zeta_{\ell}^{j}\right)=0 .
$$

Also, $\operatorname{Tr}_{0}\left(\zeta_{\ell}\right)=\frac{-1}{\ell-1}$ since the characteristic polynomial of $\zeta_{\ell}$ is $X^{\ell-1}+\cdots+X+1$, $\operatorname{deg}\left(\mathbb{Q}\left(\zeta_{\ell}\right) / \mathbb{Q}\right)=\ell-1$

Suppose that $x+w y \in \mathscr{O}_{K}$ is such that $N_{K / \mathbb{Q}}(x+w y)=p^{h}$. Here, $w=\sqrt{-d}$ if $d \equiv 1,2$ $(\bmod 4), w=\frac{1+\sqrt{-d}}{2}$ if $d \equiv 3(\bmod 4)$. Then $x, x+\frac{y}{2}=\operatorname{Tr}_{0}(x+w y)$, respectively. In all cases, we found such $Z$ represented as a ratio of Gauss sums, which can be written as $\sum_{\zeta \in S} \zeta$, where $S$ is an explicitly given set of $m$-th roots of unity (or 6-th roots of unity if $d=3)$. Thus, $x, x+\frac{1}{2} y$ can be computed as

$$
\operatorname{Tr}_{0}(Z)=\operatorname{Tr}_{0}\left(\sum_{\zeta \in S} \zeta\right)=\sum_{\zeta \in S} \operatorname{Tr}_{0}(\zeta)
$$

Suppose $\zeta \neq 1$ is a root of unity, $n=\operatorname{order}(\zeta)$. Then

$$
\prod_{\xi^{n}=1, \xi \neq 1}(1-\xi)=\left.\left(\frac{X^{n}-1}{X-1}\right)\right|_{X=1}=n
$$

so $(1-\zeta)$ divides $n$ in $\mathscr{O}_{\mathbb{Q}(\zeta)}$.

In particular, if a prime $p$ does not divide $m(p \not \backslash 6$ if $d=3)$, then any prime $\wp$ of $L=\mathbb{Q}\left(\zeta_{m}\right)$ above $p$ does not divide $(1-\zeta)$ if $\zeta \neq 1$ and $\zeta$ is an $m$-th root of unity (or 6 -th root of unity if $d=3$ ) in $L$. Therefore, if $\mathbb{F}_{q}$ is the residue field of $\wp$, then the map 
$\mu_{m} \rightarrow \mathbb{F}_{q}^{\times}\left(\mu_{6} \rightarrow \mathbb{F}_{q}\right.$ if $\left.d=3\right)$ is injective, so $\operatorname{Tr}_{0}(\zeta)=t(\zeta(\bmod \wp))$ and

$$
\operatorname{Tr}_{0}(Z)=\sum_{\zeta \in S} t(\zeta \quad(\bmod \wp))
$$

We will now write such formulas explicitly. We extend $t: \mathbb{F}_{q}^{\times} \rightarrow \mathbb{Q}$ to $\mathbb{F}_{q}$ by setting $t(0)=0$.

The case $d=1$ : Here, $Z=J\left(\chi_{1}, \chi_{2}\right)$ with $\chi_{1}=\omega^{\frac{p-1}{2}}, \chi_{2}=\omega^{\frac{p-1}{4}}$. Then for $p \equiv 1$ $(\bmod 4)$

$$
\begin{aligned}
x & =\operatorname{Tr}_{0}\left(\sum_{a \in \mathbb{F}_{q}, a \neq 0,1} \chi_{1}(a) \chi_{2}(1-a)\right)=\sum_{a \in \mathbb{F}_{q}, a \neq 0,1}\left(\frac{a}{p}\right) \operatorname{Tr}_{0}\left(\chi_{2}(1-a)\right)= \\
& =\sum_{a \in \mathbb{F}_{q}, a \neq 0,1}\left(\frac{a}{p}\right) t\left((1-a)^{\frac{p-1}{4}}\right) .
\end{aligned}
$$

Here, $\left(\frac{a}{p}\right)=1$ if $a$ is a square in $\mathbb{F}_{p},\left(\frac{a}{p}\right)=-1$ otherwise.

The case $d=3, p \equiv 1(\bmod 3)$ : Here, $Z=J\left(\chi_{1}, \chi_{2}\right)$ with $\chi_{1}=\omega^{\frac{p-1}{6}}, \chi_{2}=\omega^{\frac{p-1}{3}}$ so

$$
x+\frac{1}{2} y=\operatorname{Tr}_{0}\left(\sum_{a \in \mathbb{F}_{p}} \chi_{1}(a) \chi_{2}(1-a)\right)=\sum_{a \in \mathbb{F}_{p}} t\left(a^{\frac{p-1}{6}}(1-a)^{\frac{p-1}{3}}\right) .
$$

The case $d=2, p \equiv 1(\bmod 8)$ : Here, $Z=J\left(\chi_{1}, \chi_{2}\right)$ with $\chi_{1}=\omega^{\frac{p-1}{8}}, \chi_{2}=\omega^{\frac{3 p-3}{8}}$, so

$$
x=\operatorname{Tr}_{0}\left(\sum_{a \in \mathbb{F}_{p}} \chi_{1}(a) \chi_{2}(1-a)\right)=\sum_{a \in \mathbb{F}_{p}} t\left(a^{\frac{p-1}{8}}(1-a)^{\frac{3 p-3}{8}}\right) .
$$

If $d=2, p \equiv 3(\bmod 8)$, then $\mathbb{F}_{q}=\mathbb{F}_{p^{2}}, \chi=\omega^{\frac{p^{2}-1}{8}}, \psi=\left.\chi\right|_{\mathbb{F}_{p}}, Z=\frac{\tau_{p}(\chi)}{\tau_{p}(\psi)}=$ 
$\sum_{a \in \mathbb{F}_{p^{2}}, \operatorname{Tr}(a)=1} \chi(a)$. Thus,

$$
x=\operatorname{Tr}_{0}\left(\sum_{a \in \mathbb{F}_{p^{2}}, \operatorname{Tr}(a)=1} \chi(a)\right)=\sum_{a \in \mathbb{F}_{p^{2}}, \operatorname{Tr}(a)=1} t\left(a^{\frac{p^{2}-1}{8}}\right) .
$$

If $d \neq 1,2,3, p$ splits in $K=\mathbb{Q}(\sqrt{-d})$, except the case $p=5$ for $d=11$, then $Z$ is defined as follows. Let $H \subset(\mathbb{Z} / m \mathbb{Z})^{\times}$be the kernel of $\chi_{d}$. Let $R=\left\{b_{i}: i=1,2, \ldots, n\right\}$ be the set of integers in the interval $[0, m)$ representing the elements of $H /\left\{1, p, \ldots, p^{f-1}\right\}$, where $f$ is the period of $p(\bmod m)$. In particular, $n=\frac{\varphi(m)}{2 f}$. Let $\chi=\omega^{\frac{q-1}{m}}: \mathbb{F}_{p^{f}}^{\times} \rightarrow \mu_{m}$, $\chi_{i}=\chi^{b_{i}}, q=p^{f}, \psi=\left.\left(\prod \chi_{i}\right)\right|_{\mathbb{F}_{p}}$. We proved in section 3.4 that $\psi=\psi_{0}$. Recall that $\eta=\frac{1}{2}\left(\frac{\varphi(m)}{2}-h\right)=\frac{\sum_{a \in H}[a]_{m}}{m} \in \mathbb{N}$.

From the results of section 3.4 , we can set

$$
Z=\frac{\prod_{i=1}^{n} \tau_{p}\left(\chi_{i}\right)}{p^{\eta}}
$$

Therefore, by property 5 on page 10 ,

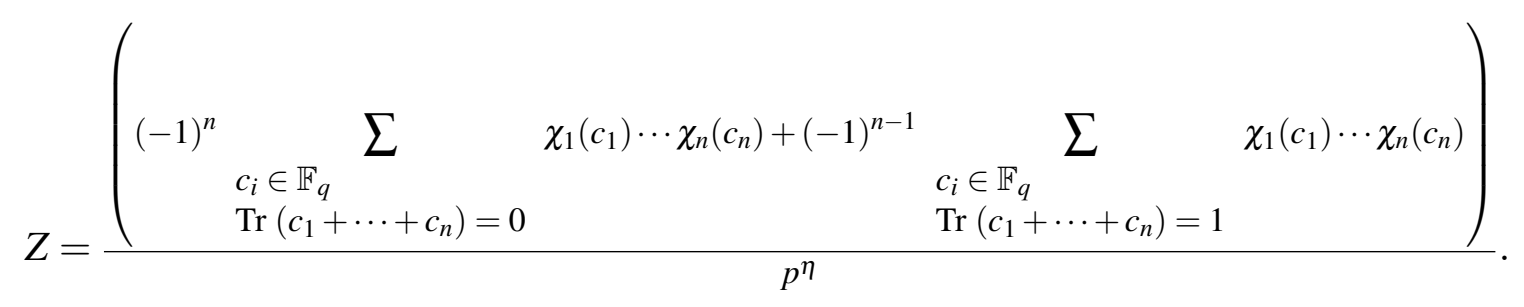


So when $d \equiv 3(\bmod 4)$ or $d \equiv 1,2(\bmod 4)$,

$$
\begin{aligned}
& x+\frac{y}{2} \text { or } x=\operatorname{Tr}_{0}(Z)
\end{aligned}
$$

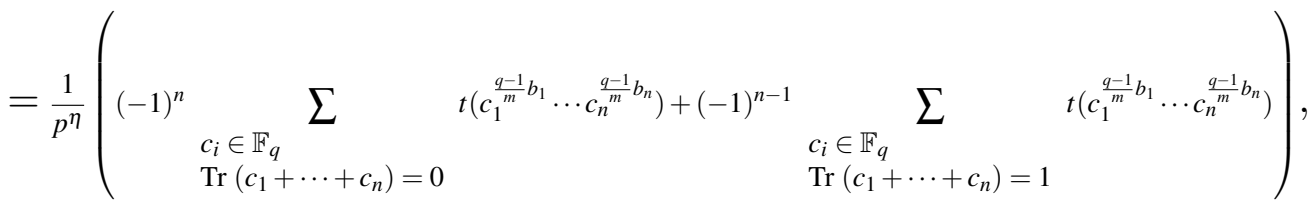

respectively.

\subsection{Examples}

With the aid of Mathematica, we compute some examples.

Example 3.1. Let $d=7$ and $p=29$. Then $h=1, T=25$ and $2 x+y=$

$$
\left\langle\Gamma_{29}\left(\frac{1}{7}\right) \Gamma_{29}\left(\frac{2}{7}\right) \Gamma_{29}\left(\frac{4}{7}\right)\right\rangle=2 \text { and } y= \pm \sqrt{\frac{4(29)-2^{2}}{7}}= \pm 4 \text {. }
$$

Example 3.2. Let $d=11$, so $h=1$. Let $p=23$. We have $T=21,2 x+y=9$ and $y=$ $\pm \sqrt{\frac{4(23)-9^{2}}{11}}= \pm 1$.

Example 3.3. Let $d=15$, so $h=2$. Let $p=31$. We have $T=897,2 x+y=-2$ and $y=$ $\pm \sqrt{\frac{4(31)^{2}-(-2)^{2}}{15}}= \pm 16$.

Example 3.4. Let $d=19$ and $p=191$ so $h=1$ and $T=181$. We have $2 x+y=17$ and $y= \pm \sqrt{\frac{4(191)-17^{2}}{19}}= \pm 5$.

Example 3.5. Let $d=23$ and $p=47$. Then $h=3$ and $c=k=2$ in Proposition 3.7. We have $T=\left[\frac{1}{23}\right]_{47^{2}}=2113,2 x+y=-48$ and $y= \pm \sqrt{\frac{4(47)^{3}-(-48)^{2}}{23}}= \pm 134$. 
Example 3.6. Let $d=23$ and $p=2$. We have $h=3$. By Proposition 3.7, $c=\frac{h+1}{2}+1=3$. We have $T=\left[\frac{1}{23}\right]_{2^{3}}=7,2 x+y=3$ and $y= \pm \sqrt{\frac{4\left(2^{3}\right)-3^{2}}{23}}= \pm 1$.

Example 3.7. Let $d=31$ and $p=2$. Here, $h=3$ and since $31>2^{3}\left(\frac{16-2}{4}\right), c=\frac{h+1}{2}=2$. Now, we cannot take the modulus to be $2^{2}=4$, so we choose $n=3$. We have $T=\left[\frac{1}{31}\right]_{2^{3}}=$ $7,2 x+y=1$ and $y= \pm \sqrt{\frac{4\left(2^{3}\right)-1^{2}}{31}}= \pm 1$.

Example 3.8. Let $d=39$ and $p=2$. Then $h=4$ and by Proposition 3.7, $c=4$. We have $T=\left[\frac{1}{39}\right]_{2^{4}}=7,2 x+y=-5$ and $y= \pm \sqrt{\frac{4\left(2^{4}\right)-(-5)^{2}}{39}}= \pm 1$.

Example 3.9. Let $d=43$ and $p=173$. We have $h=1$ and $T=169,2 x+y=-2$ and $y= \pm \sqrt{\frac{4(173)-(-2)^{2}}{43}}= \pm 4$.

Example 3.10. Suppose $d=51$. Then $h=2$. Let $p=103$. Then $T=10,401$. We have $2 x+y=-155$ and $y= \pm \sqrt{\frac{4(103)^{2}-(-155)^{2}}{51}}= \pm 19$.

Example 3.11. Suppose $d=119$ and $p=2$. Then $h=10$. By Proposition 3.7, we have $c=\frac{10}{2}+1+1=7$ so that $T=\left[\frac{1}{119}\right]_{2^{7}}=71,2 x+y=-55$ and $y= \pm \sqrt{\frac{4\left(2^{10}\right)-(-55)^{2}}{119}}= \pm 3$.

Example 3.12. Let $d=191$ and $p=2$. Then $h=13, c=\frac{13+1}{2}+2=9$. We have $T=$ $\left[\frac{1}{191}\right]_{2^{9}}=319,2 x+y=153, y= \pm \sqrt{\frac{4\left(2^{13}\right)-153^{2}}{191}}= \pm 7$.

Example 3.13. Let $d=327$ and $p=2$. We have $h=12$ and $c=\frac{12}{2}+1+1=8$. We get $T=\left[\frac{1}{327}\right]_{2^{8}}=119,2 x+y=19$ so that $y= \pm \sqrt{\frac{4\left(2^{12}\right)-19^{2}}{327}}= \pm 7$. 
Example 3.14. Let $d=6$, then $h=2$. Let $p=73$. We have $T=5107$, $x=$ $\left\langle\frac{1}{2} \Gamma_{73}\left(\frac{1}{24}\right) \Gamma_{73}\left(\frac{5}{24}\right) \Gamma_{73}\left(\frac{7}{24}\right) \Gamma_{73}\left(\frac{11}{24}\right)\right\rangle=-25$ and $y= \pm \sqrt{\frac{73^{2}-(-25)^{2}}{6}}= \pm 28$.

Example 3.15. Let $d=21$ so that $h=4$ and $c=k=3$ in Proposition 3.7. Let $p=337$.

Then $T=37,817,125$,

$x=\left\langle\frac{1}{2} \Gamma_{337}\left(\frac{1}{84}\right) \Gamma_{337}\left(\frac{5}{84}\right) \Gamma_{337}\left(\frac{11}{84}\right) \Gamma_{337}\left(\frac{17}{84}\right) \Gamma_{337}\left(\frac{19}{84}\right) \Gamma_{337}\left(\frac{23}{84}\right) \Gamma_{337}\left(\frac{25}{84}\right) \Gamma_{337}\left(\frac{31}{84}\right) \Gamma_{337}\left(\frac{37}{84}\right) \Gamma_{337}\left(\frac{41}{84}\right) \Gamma_{337}\left(\frac{55}{84}\right) \Gamma_{337}\left(\frac{71}{84}\right)\right\rangle$

$=110,881$ and $y= \pm \sqrt{\frac{337^{4}-(110,881)^{2}}{21}}= \pm 5,360$. 


\section{Chapter 4}

\section{Finding the Height of the Stickelberger Ideal}

Let $\zeta_{m}$ be a primitive $m$-th root of unity, $M / \mathbb{Q}$ be a finite abelian extension, $G=\operatorname{Gal}(M / \mathbb{Q})$ and $G_{m}=\operatorname{Gal}\left(\mathbb{Q}\left(\zeta_{m}\right) / \mathbb{Q}\right)$. For $(a, m)=1$, let $\sigma_{a} \in G_{m}$ denote the element such that $\zeta_{m} \mapsto \zeta_{m}^{a}$ as well as its restriction to $M$. Let $I=I(M)$ be the Stickelberger ideal (see section 2.1).

Case 1: $M=\mathbb{Q}\left(\zeta_{m}\right)$

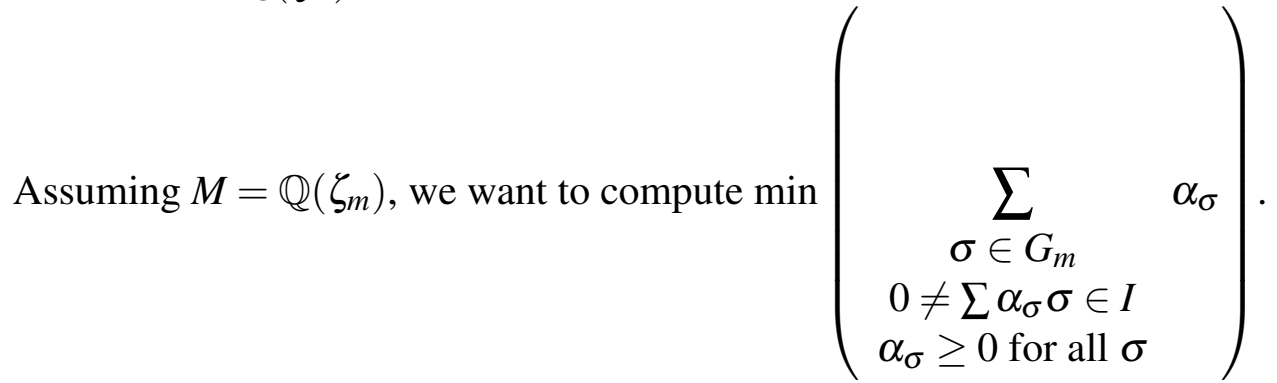

We first note that the map $\phi: \sum \alpha_{\sigma} \sigma \mapsto \sum \alpha_{\sigma}$ is a homomorphism. Now let $\sum x_{i} \sigma_{i} \in$ $\mathbb{Z}[G] \backslash\{0\}$ such that $\left(\sum x_{i} \sigma_{i}\right) \theta \in \mathbb{Z}[G]$. That is,

$$
\left(\sum_{i} x_{i} \sigma_{i}\right)\left(\sum_{b} \frac{b}{m} \sigma_{b^{-1}}\right)=\sum_{b} \sum_{i} \frac{x_{i} b}{m} \sigma_{i b^{-1}}=\sum_{a} \sum_{i} \frac{i a x_{i}}{m} \sigma_{a^{-1}} \in \mathbb{Z}[G],
$$


where $a^{-1} \equiv i b^{-1}(\bmod m)$. By the homomorphism $\phi$, we get

$$
\left(\sum_{i} x_{i}\right)\left(\sum_{b} \frac{b}{m}\right)=\sum_{a} \sum_{i} \frac{i a x_{i}}{m} \in \mathbb{Z}
$$

Since

$$
\sum_{\substack{1 \leq b<m \\(b, m)=1}} \frac{b}{m}=\frac{m \cdot \varphi(m) / 2}{m}=\frac{\varphi(m)}{2}
$$

equation (4.1) is equivalent to

$$
\left(\sum_{i} x_{i}\right) \frac{\varphi(m)}{2}=\sum_{a} \sum_{i} \frac{i a x_{i}}{m} \in \mathbb{Z}
$$

Since we want to consider only cases where the coefficients are nonnegative, this means that $\sum_{i} x_{i} \in \mathbb{Z}^{+}$. That is, $\min \left(\sum_{a} \sum_{i} \frac{\operatorname{iax}_{i}}{m}\right) \geq \frac{\varphi(m)}{2}$. To determine the actual minimum, we look at two cases:

The first case is $(2, m)=1$. In this case, we have

$$
\left(2-\sigma_{2}\right) \theta=\left(2-\sigma_{2}\right) \sum_{\substack{1 \leq a<m \\(a, m)=1}} \frac{a}{m} \sigma_{a}^{-1}=\sum_{\substack{1 \leq a<m \\(a, m)=1}} \frac{2 a}{m} \sigma_{a^{-1}}-\sum_{\substack{1 \leq a<m \\(a, m)=1}} \frac{a}{m} \sigma_{2 a^{-1}} .
$$

Let $b^{-1} \equiv 2 a^{-1}(\bmod m)$ and write $[b]_{m}=[b]$ to mean the integer in $[0, m)$ representing $b$ 
$(\bmod m)$. Thus, equation $(4.3)$ equals

$$
\begin{aligned}
& \sum_{1 \leq a<m} \frac{2 a}{m} \sigma_{a^{-1}}-\sum_{1 \leq b<m} \frac{[2 b]}{m} \sigma_{b^{-1}}=\sum_{1 \leq b<m} \frac{2 b-[2 b]}{m} \sigma_{b^{-1}} \\
& (a, m)=1 \quad(b, m)=1 \quad(b, m)=1 \\
& =\sum_{1 \leq b<m}\left\lfloor\frac{2 b}{m}\right\rfloor \sigma_{b^{-1}} \\
& (b, m)=1
\end{aligned}
$$

where $\lfloor x\rfloor$ denotes the greatest integer function.

For $2 b<m,\left\lfloor\frac{2 b}{m}\right\rfloor=0$. For $2 b>m$, we note that since we are summing values of $b$ for which $b<m$, then $2 b<2 m$, so that $\left\lfloor\frac{2 b}{m}\right\rfloor=1$. This means that the summation (4.5) is equivalent to

$$
\begin{aligned}
& \sum_{\frac{m}{2}<b<m} \sigma_{b}^{-1} \\
& (b, m)=1
\end{aligned}
$$

Hence, we want to look at $\sum_{\substack{\frac{m}{2}<b<m \\(b, m)=1}} 1$. We consider the question of how many $b$ 's satisfy $m<2 b$ and $(b, m)=1$. The residues relatively prime to $m$ are distributed symmetrically about $\frac{m}{2}$, so there are $\frac{\varphi(m)}{2}$ many such $b$ 's. Therefore, $\sum_{\substack{\frac{m}{2}<b<m \\(b, m)=1}} 1=\frac{\varphi(m)}{2}$.

We have shown that there is an element in the Stickelberger ideal such that the sum of the coefficients is equal to $\frac{\varphi(m)}{2}$. That is, when $(m, 2)=1$, we have 
$\min \left(\begin{array}{c}\sum_{\sigma \in G_{m}} \alpha_{\sigma} \\ 0 \neq \sum \alpha_{\sigma} \sigma \in I \\ \alpha_{\sigma} \geq 0 \text { for all } \sigma\end{array}\right)=\frac{\varphi(m)}{2}$

For the case $(m, 2) \neq 1$, we claim that $\min$

$$
\left(\sum_{\substack{\sigma \in G_{m} \\ 0 \neq \sum \alpha_{\sigma} \sigma \in I \\ \alpha_{\sigma} \geq 0 \text { for all } \sigma}} \alpha_{\sigma}\right)=\varphi(m) \text {. To see }
$$

that there exists an element in the Stickelberger ideal such that the sum of coefficients is equal to $\varphi(m)$, consider

$$
\begin{aligned}
\left(\sigma_{1}+\sigma_{m-1}\right) \theta & =\left(\sigma_{1}+\sigma_{-1}\right)\left(\sum_{b} \frac{b}{m} \sigma_{b^{-1}}\right)=\sum_{b} \frac{b}{m} \sigma_{b^{-1}}+\sum_{b} \frac{b}{m} \sigma_{-b^{-1}} \\
& =\sum_{b} \frac{b}{m} \sigma_{b^{-1}}+\sum_{a} \frac{m-a}{m} \sigma_{a^{-1}}=\sum_{b} \sigma_{b^{-1}}
\end{aligned}
$$

This element of the Stickelberger ideal has $\sum_{b} 1=\varphi(m)$.

Now, to see that there are no elements in the Stickelberger ideal with $\sum \alpha_{\sigma}=\frac{\varphi(m)}{2}$, we assume there is one and obtain a contradiction. From equation (4.2), we have $\left(\sum_{i} x_{i}\right) \frac{\varphi(m)}{2}=\sum_{a} \sum_{i} \frac{i a x_{i}}{m} \in \mathbb{Z}$. This sum being equal to $\frac{\varphi(m)}{2}$ implies that $\sum_{i} x_{i}=1$. On the other hand, $\sum_{a} \sum_{i} \frac{i a x_{i}}{m} \sigma_{a^{-}} \in \mathbb{Z}[G]$ tells us that $\sum_{i} \frac{i a x_{i}}{m} \in \mathbb{Z}$, or $\sum_{i} i a x_{i} \equiv 0(\bmod m)$. Since $(m, 2) \neq 1$ and $(i, m)=(a, m)=1$, this tells us that $\sum_{i} x_{i} \equiv 0(\bmod 2)$, which contradicts $\sum_{i} x_{i}=1$. Thus, there are no elements in the Stickelberger ideal such that 
$\sum \alpha_{\sigma}=\frac{\varphi(m)}{2}$ for the case $(m, 2) \neq 1$. Since there is an element with $\sum x_{i}=2$ (equivalently, $\left.\sum \alpha_{\sigma}=\varphi(m)\right)$, we see that $\min$

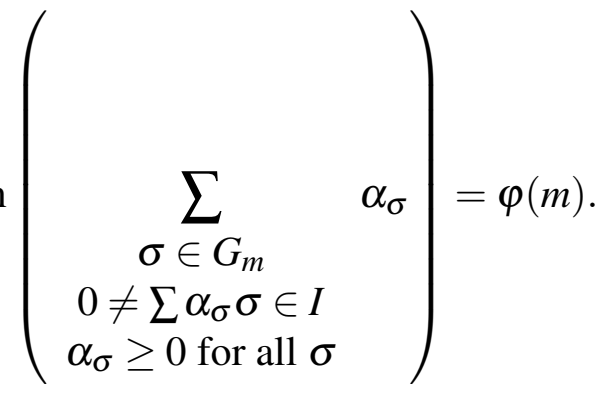

Case 2: $M \subset \mathbb{Q}\left(\zeta_{m}\right)$.

More generally, we will assume that $M$ is a proper subfield of $\mathbb{Q}\left(\zeta_{m}\right)$. The result follows almost immediately from the above. For the case $(2, m)=1$, we see that $\left(2-\sigma_{2}\right) \theta \in$ $\mathbb{Z}\left[G_{m}\right]$ actually implies $\left(2-\sigma_{2}\right) \theta \in \mathbb{Z}[G]$, where $G=\operatorname{Gal}(M / \mathbb{Q}) \cong \frac{G_{m}}{\operatorname{Gal}\left(\mathbb{Q}\left(\zeta_{m}\right) / M\right)}$. Note that when we pass to the quotient, the coefficients of elements in $G$ will be sums of coefficients of elements in $G_{m}$, and thus will be in $\mathbb{Z}$ also. Hence, the minimum will again be $\frac{\varphi(m)}{2}$. Similarly, for $(2, m) \neq 1,\left(\sigma_{1}+\sigma_{m-1}\right) \theta \in \mathbb{Z}\left[G_{m}\right]$ implies $\left(\sigma_{1}+\sigma_{m-1}\right) \theta \in \mathbb{Z}[G]$ and the minimum of the sum of the coefficients will be $\varphi(m)$. 


\section{Bibliography}

[1] Z. I. Borevich and I. R. Shafarevich, Number Theory, Academic Press, 1966.

[2] Henri Cohen, Number Theory, vol. II: Analytic and Modern Tools, Springer-Verlag, 2007.

[3] _ Number Theory, vol. I: Tools and Diophantine Equations, Springer-Verlag, 2007.

[4] Benedict H. Gross and Neal Koblitz, Gauss sums and the p-adic $\Gamma$-function, Annals of Mathematics 109 (1979), no. 3, 569-581.

[5] Kenneth Ireland and Michael Rosen, A classical Introduction to Modern Number Theory, 2nd ed., Springer-Verlag, 2010.

[6] Gerald J. Janusz, Algebraic Number Fields, 2nd ed., American Mathematical Society, 1996.

[7] S. A. Katre, Gauss-Jacobi sums and Stickelberger's Theorem, Cyclotomic fields and related topics (2000), 75-92.

[8] Neal Koblitz, p-adic Numbers, p-adic Analysis, and Zeta-Functions, 2nd ed., Springer, 1984.

[9] Serg Lang, Cyclotomic Fields I and II, combined 2nd ed., Springer-Verlag, 1990.

[10] _ Algebraic Number Theory, 2nd ed., Springer-Verlag, 1994.

[11] Daniel A. Marcus, Number Fields, Springer, 1977.

[12] Jürgen Neukirch, Algebraic Number Theory, Springer-Verlag, 1999.

[13] Alain M. Robert, The Gross-Koblitz Formula Revisited, Rendiconti del Seminario Matematico della Università di Padova 105 (2001), 157-170. 
[14] Lawrence C. Washington, Introduction to Cyclotomic Fields, 2nd ed., Springer, 1997. 